\title{
CONVERGENCE OF A CANCER INVASION MODEL TO A LOGISTIC CHEMOTAXIS MODEL
}

\author{
THOMAS HILLEN \\ Center for Mathematical Biology, \\ Department of Mathematical and Statistical Sciences, \\ University of Alberta, Edmonton T6G 2G1, Canada \\ thillen@ualberta.ca
}

\section{KEVIN J. PAINTER}

Department of Mathematics and Maxwell Institute for Mathematical Sciences, Heriot-Watt University, Edinburgh EH14 4AS, UK

K.Painter@hw.ac.uk

\section{MICHAEL WINKLER}

Institut für Mathematik, Universität Paderborn, 33098 Paderborn, Germany

michael.winkler@uni-due.de

Received 5 December 2011

Revised 6 March 2012

Accepted 25 March 2012

Published 5 September 2012

Communicated by P. Maini

\begin{abstract}
A characteristic feature of tumor invasion is the destruction of the healthy tissue surrounding it. Open space is generated, which invasive tumor cells can move into. One such mechanism is the urokinase plasminogen system (uPS), which is found in many processes of tissue reorganization. Lolas, Chaplain and collaborators have developed a series of mathematical models for the uPS and tumor invasion. These models are based upon degradation of the extracellular material through plasmid plus chemotaxis and haptotaxis. In this paper we consider the uPS invasion models in one-space dimension and we identify a condition under which this cancer invasion model converges to a chemotaxis model with logistic growth. This condition assumes that the density of the extracellular material is not too large. Our result shows that the complicated spatiotemporal patterns, which were observed by Lolas and Chaplain et al. are organized by the chaotic attractor of the logistic chemotaxis system. Our methods are based on energy estimates, where, for convergence, we needed to find lower estimates in $L^{\gamma}$ for $0<\gamma<1$. This is a new method for these types of PDE.
\end{abstract}

Keywords: Chemotaxis; haptotaxis; cancer invasion.

AMS Subject Classification: 35B33, 35B45, 35K55, 35K57, 92C17 


\section{Introduction}

Tumor development and progression is a highly complex process characterized by the accumulation of various hallmark features including abnormal growth, genetic instabilities, interactions with the micro-environment and immune system, invasion and metastasis. ${ }^{5}$ The latter in particular, invasion and metastasis, is associated with a grave prognosis for the patient. Mathematical modeling of cancer has emerged into a large discipline, with models developed to describe these many facets of cancer development, aiming to shed fresh impetus on tumor formation, progression and treatment.

In this paper we focus on a specific model for tumor invasion, developed by Chaplain and coworkers over a series of papers $^{2-4}$ and based on the urokinase plasminogen system. The urokinase plasminogen system is considered as a prototypical chemical network playing a key role in many biological processes where significant remodeling of the extracellular matrix (ECM) occurs, for example wound healing, embryonic development and cancer invasion. A key component is plasmin, which degrades the ECM and permits cancer cells to break free from the main tumor mass and invade new areas. Five of the major players in the urokinase plasminogen system are the urokinase plasminogen activator $\mathrm{uPA}$, the urokinase plasminogen receptor $\mathrm{uPAR}$, the plasminogen activator inhibitor PAI-1, plasmin and the ECM material vitronectin VN, the latter a glue-like substance found in the ECM. uPA activates plasminogen, which creates plasmin, a proteolytic enzyme which is able to degrade ECM. Invasive cancer cells are equipped with uPA receptors, uPAR, which both bind to focal adhesion points, thereby anchoring the cell to the ECM (in particular to $\mathrm{VN}$ ), as well as binding uPA and stimulating the local release of plasmin at the leading edge of the moving cell. The plasmin degrades ECM at the leading edge, creating the space for a cell to move into. The inhibitor PAI-1 inhibits plasmin production and, in high enough concentrations, can halt ECM degradation. In low concentrations, however, it can facilitate cell movement through detaching the cell-ECM adhesions at the cell rear, thereby releasing the brakes and allowing movement forward. A more detailed description of the above described uPA dynamics can be found in Ref. 3 .

The above dynamics were incorporated by Chaplain and Lolas ${ }^{3}$ into a reactionadvection-diffusion equation system composed of five variables: an equation for the cancer cells that describes their growth, random, chemotactic and haptotactic movement and variables for the ECM, uPA, PAI-1 and plasmin. In simulations a front of cells invading into the ECM was observed, followed by complex spatiotemporal dynamics suggested to arise from "the complex interplay between proliferative effects - cancer cell proliferation and matrix remodelling - and gradient driven migration (chemotaxis and haptotaxis)" (Chaplain and Lolas, ${ }^{4}$ p. 1726). Chaplain and Lolas argue that there is a conceptual similarity between the irregular patterns from the model and certain forms of in vivo tumor morphology, which can be irregular in shape and form with tumor-free regions separating tumor micro-colonies. 
The origin of the complex dynamics is the motivation for the present study where, in fact, we find that they are fundamentally driven by cell proliferation and chemotaxis with ECM remodeling and haptotaxis playing only a minor role. In Ref. 4 a subsystem composed of three equations was studied in more depth, given in (1.1) below and focusing on the dynamics between cancer cells, $u(x, t)$, uPA, $v(x, t)$ and the ECM, $w(x, t)$. Chaplain and Lolas study the invasion patterns, but they do not explore complex spatio-temporal patterns for this model; here we will show that this three-component model also possesses spatio-temporal dynamics. Andasari et al. ${ }^{2}$ study the original five-component model in greater detail, computing the unstable modes and determining a relationship between model parameters and observed patterns.

The patterns shown by the models described above bear a close superficial resemblance to the spatio-temporal chaos found for chemotaxis models by Painter and Hillen, ${ }^{12}$ suggesting a close correlation between the models. Here we will demonstrate that the three-component model does in fact, in an appropriate sense, converge to the chemotaxis model with logistic growth studied by Painter and Hillen ${ }^{12}$ under certain conditions and, since the chemotaxis model with logistic growth has a compact global attractor, ${ }^{11,1}$ we can conclude that this attractor is the organizing center for the complex dynamics of the three-component urokinase plasminogen system. While we suspect that the same mechanisms are the driving force behind the complex dynamics of the five-component system of Lolas and Chaplain, ${ }^{3} \mathrm{a}$ rigorous proof has not yet been obtained.

\subsection{The models}

In the spatially one-dimensional framework, the three-component urokinase plasminogen invasion model from Lolas and Chaplain (which we call the cancer invasion model, for short) is given as

$$
\left\{\begin{array}{lll}
u_{t}=D_{1} u_{x x}-\chi\left(u v_{x}\right)_{x}-\xi\left(u w_{x}\right)_{x}+r u-\mu u^{2}-\lambda u w, & x \in \Omega, & t>0, \\
v_{t}=D_{2} v_{x x}-a v+b u, & x \in \Omega, & t>0, \\
w_{t}=-\rho v w, & x \in \Omega, & t>0, \\
u_{x}(x, t)=v_{x}(x, t)=0, & x \in \partial \Omega, & t>0, \\
u(x, 0)=u_{0}(x), \quad v(x, 0)=v_{0}(x), & w(x, 0)=w_{0}(x), & x \in \Omega,
\end{array}\right.
$$

where $\Omega$ is a bounded real interval. The parameters $D_{1}, D_{2}, \chi, \xi, r, \mu, a, b$ and $\rho$ are supposed to be positive and $\lambda$ to be non-negative. The variables $(u, v, w)$ describe the densities or concentrations of tumor cells, uPA and ECM, respectively. The initial data $u_{0} \in C^{0}(\bar{\Omega}), v_{0} \in W^{1, \infty}(\Omega)$ and $w_{0} \in C^{1}(\bar{\Omega})$ are prescribed non-negative functions.

It is noted that the original model by Chaplain and Lolas ${ }^{3}$ has an additional term to describe the remodeling of the healthy tissue; in that case the equation for 
$w$ reads

$$
w_{t}=-\rho v w+\mu_{2} w(1-w),
$$

with some $\mu_{2}>0$. In their simulation examples the value for $\mu_{2}$ is one to two orders of magnitude smaller than $\rho$ and, for our study, we have assumed that there is no regeneration of ECM, i.e. $\mu_{2}=0$. This assumption is crucial for our results and allows us to show that system (1.1) can have chaotic dynamics. We perform some simulations for nonzero $\mu_{2}$ later, showing that at least for small $\mu_{2}$ the dynamics are essentially the same. However a rigorous analytical result for the case of $\mu_{2} \neq 0$ is left for future investigations.

The associated chemotaxis model with logistic growth reads

$$
\left\{\begin{array}{lll}
U_{t}=D_{1} U_{x x}-\chi\left(U V_{x}\right)_{x}+r U-\mu U^{2}, & x \in \Omega, & t>0, \\
V_{t}=D_{2} V_{x x}-a V+b U, & x \in \Omega, & t>0, \\
U_{x}(x, t)=V_{x}(x, t)=0, & x \in \partial \Omega, & t>0,
\end{array}\right.
$$

with corresponding initial conditions for $U$ and $V$. An overview about chemotaxis modeling with PDEs can be found in Ref. 6 .

In Fig. 1 we show simulations of these models (1.1) and (1.2) for the parameter choices $D_{1}=D_{2}=\lambda=r=\mu=a=b=\rho=1$ and $\xi=10$, with varying values of $\chi$. In the first row (a) we choose $\chi=5$ and in the second row (b) $\chi=15$. The first three frames (a1)-(a3) and (b1)-(b3) show the solution $(u, v, w)$ of the cancer invasion model (1.1) and frames (a4), (a.5) and (b4), (b5) show the solution $(U, V)$ of the chemotaxis model (1.2). For the plasminogen model (a1)-(a3) and (b1)-(b3), we see an invasion front which degrades the ECM, leaving either a stationary (case (a)) or evolving (case (b)) pattern of cancer cell micro-aggregates in its wake. The patterns of the corresponding chemotaxis model in (a4), (a5) and (b4), (b5) are basically identical to those that eventually arise from the cancer invasion model despite the distinct initial behavior. Painter and Hillen ${ }^{12}$ studied these patterns for the chemotaxis model in detail and identified a mechanism of merging and emerging of local peaks, showing that for certain regions of parameter space the dynamics are chaotic. Consequently we expect the same to be true for the cancer invasion model.

\subsection{Main results}

The current literature provides only a few results on the qualitative behavior in models of type (1.1). This is in sharp contrast to the chemotaxis-growth system (1.2) and its $n$-dimensional analogue; indeed, it is known that solutions to the latter remain bounded when either $n \leq 2,{ }^{11}$ or when $n \geq 3$ and $\mu$ is large. ${ }^{20}$ In the case where $n=2$ more detailed information is available on their dynamics, including the existence of global and exponential attractors and their dimensions. ${ }^{11,10}$ The crucial mathematical novelty in (1.1) consists of the interaction with the non-diffusive ECM component $w$. As observed in previous studies on haptotaxis-only systems, this may 


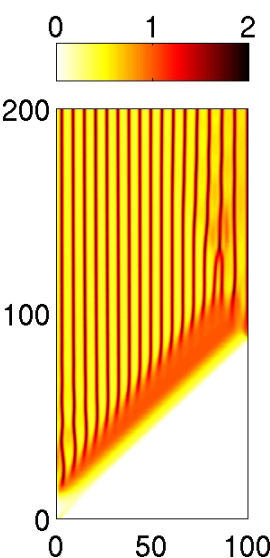

(a1)

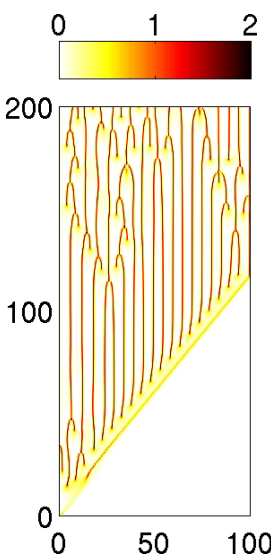

(b1)
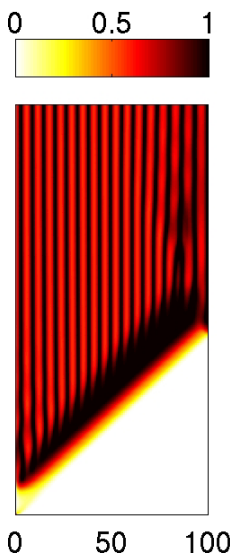

(a2)


(b2)


50

(a3)
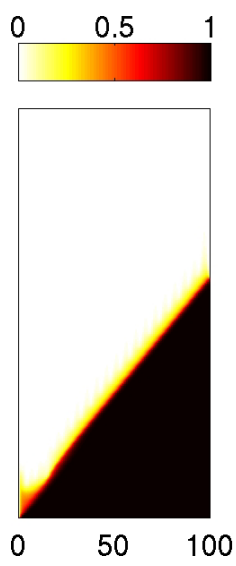

(b3)
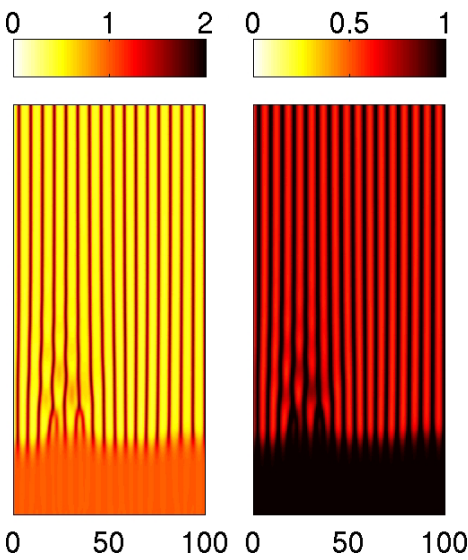

(a4)

(a5)
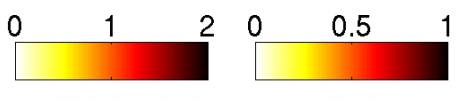

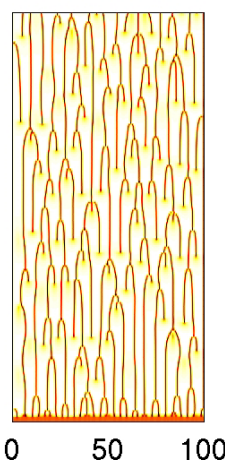

(b4)

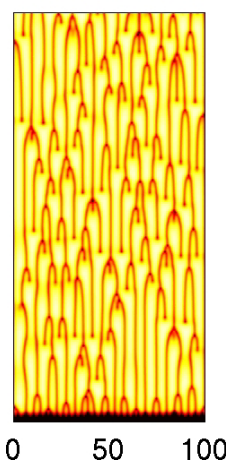

(b5)

Fig. 1. (Color online) Numerical simulations comparing the spatio-temporal evolution of the plasminogen model (1.1) with the chemotaxis model (1.2). Each frame plots the space (horizontal)time (vertical) density map, using the color scale at the top of each frame. The parameter values are $D_{1}=D_{2}=\lambda=r=\mu=a=b=\rho=1$ and $\xi=10$, with $\chi=5$ in the first row (a), and $\chi=15$ in the second row (b). Figures (a1)-(a3) and (b1)-(b3) show the cancer invasion model (1.1) plotting the cell density $u$ in (a1), (b1), the chemoattractant $v$ in (a2), (b2) and the ECM $w$ in (a3), (b3). Figures (a4), (a5) and (b4), (b5) show the corresponding behavior of the chemotaxis model (1.2) for the cell density $u$ in (a4), (b4) and the chemoattractant $v$ in (a5), (b5). The initial conditions were set at $u_{0}(x)=v_{0}(x)=\exp \left(-x^{2}\right)$ and $w_{0}(x)=1-u_{0}(x)$ for the cancer model, and $u_{0}(x)=1, v(x, 0)=1+\epsilon(x)$ (where $\epsilon(x)$ denotes a small random spatial perturbation) for the chemotaxis model. Numerical simulations were performed as described at the start of Sec. 6 on the spatial domain $[0,100]$ with a spatial discretization of $\Delta x=0.05$ and error tolerances of $10^{-8}$.

carry with it numerous technical obstacles in not only the existence theory, but also the qualitative study of solutions. ${ }^{9,8,17}$ Accordingly, analytical investigations into the three-component system (1.1) have concentrated to date on the global existence of solutions. Indeed, solutions are known to be global in time when either $n \leq 2$, 
or when $n=3$ and $\mu$ and $\lambda$ are large enough. This has recently been shown in a series of papers which also address some variants involving nonlinear cell diffusion, as suggested in Ref. 3 (see also Refs. 13, 15, 14, 16 and the references therein).

The first objective of the present paper is to address the boundedness question for (1.1). Our main result in this direction is the following.

Theorem 1.1. Let $\Omega \subset \mathbb{R}$ be an open bounded interval. Suppose that $D_{1}, D_{2}, \rho, a$ and $\mu$ are positive, and that $\chi, \xi, r, \lambda$ and $b$ are non-negative. Then for any nonnegative $u_{0} \in C^{0}(\bar{\Omega}), v_{0} \in W^{1, \infty}(\Omega)$ and $w_{0} \in C^{1}(\bar{\Omega})$ satisfying $w_{0 x}=0$ on $\partial \Omega$, the corresponding solution $(u, v, w)$ of $(1.1)$ is bounded in $\Omega \times(0, \infty)$.

Let us mention here that, as it stands, our proof appears to apply only to space dimension one. Thus, the boundedness question in the higher-dimensional version of (1.1) has to be left as an open problem at present.

Secondly, we shall address the question as to whether, and in which sense, the third solution component $w$ decays for large times. In that case the tumor would invade the healthy tissue and completely degrade the ECM. A linear analysis of homogeneous steady states (see Sec. 5.1) shows that the tumor can only invade if

$$
\lambda w^{*}<r
$$

where $w^{*}$ denotes the ECM density in healthy tissue. This condition is made more precise in our second result.

Theorem 1.2. Let $\lambda \geq 0$ and assume that $u_{0} \not \equiv 0$ and

$$
\lambda\left\|w_{0}\right\|_{L^{\infty}(\Omega)} \leq r .
$$

Then there exists $\theta>0$ with the property that for all $q \in[2, \infty)$ one can find $C>0$ such that

$$
\|w(\cdot, t)\|_{W^{1, q}(\Omega)} \leq C e^{-\theta t} \quad \text { for all } t>0 .
$$

Hence, in this case, all ECM is degraded and the solution of the cancer invasion model (1.1) asymptotically behaves as if the term $w$ in (1.1) was absent, that is, like a solution of the pure chemotaxis system (1.2). As shown numerically by Painter and Hillen, ${ }^{12}$ the chemotaxis model (1.2) can show chaotic dynamics. Hence in certain parameter ranges, the solutions depend sensitively on the initial conditions and we cannot assume that two solutions converge. Consequently, convergence of a single solution of the cancer invasion model (1.1) to a specific solution of the chemotaxis model (1.2) cannot be expected. However, we obtain convergence in a more global sense, which we describe through the corresponding solution operators. We can apply the variation-of-constants formula in order to represent the solution of the chemotaxis model (1.2) by using two nonlinear operators $\Phi_{1}$ and $\Phi_{2}$ according to

$$
\begin{aligned}
U(x, t) & =e^{D_{1}\left(t-t_{0}\right) \Delta} U\left(x, t_{0}\right)-\int_{t_{0}}^{t} e^{D_{1}(t-s) \Delta}\left\{\chi\left(U V_{x}\right)_{x}-r U+\mu U^{2}\right\}(\cdot, s) d s \\
& =: \Phi_{1}\left(x, t ; U, V, t_{0}\right)
\end{aligned}
$$


and

$$
\begin{aligned}
V(x, t) & =e^{D_{2}\left(t-t_{0}\right) \Delta} V\left(x, t_{0}\right)+\int_{t_{0}}^{t} e^{D_{2}(t-s) \Delta}\{b U-a V\}(\cdot, s) d s \\
& =: \Phi_{2}\left(x, t ; U, V, t_{0}\right)
\end{aligned}
$$

for arbitrary $t_{0} \geq 0$ and all $(x, t) \in \Omega \times\left(t_{0}, \infty\right)$. Using this notation, we can write our convergence result as follows.

Corollary 1.3. Let $\lambda \geq 0$ and $u_{0} \not \equiv 0$, and suppose that (1.3) holds. Then there exist $C>0$ and $\theta>0$ such that

$$
\sup _{t>t_{0}}\left\|u(\cdot, t)-\Phi_{1}\left(\cdot, t ; u, v, t_{0}\right)\right\|_{L^{\infty}(\Omega)} \leq C e^{-\theta t_{0}}
$$

and

$$
\sup _{t>t_{0}}\left\|v(\cdot, t)-\Phi_{2}\left(\cdot, t ; u, v, t_{0}\right)\right\|_{L^{\infty}(\Omega)}=0
$$

for all $t_{0}>0$.

Let us also remark that the appearance of the smallness condition (1.3) is not of a purely technical nature. Indeed, a simple counterexample shows that it cannot be relaxed (see Proposition 5.1). However, convergence can be observed numerically, even in cases where condition (1.3) is not true. In fact, as a by-product of our analysis we can identify a dichotomy. Namely, either the cancer invasion model converges to the chemotaxis model, or the total mass of cells vanishes in the large time limit.

Theorem 1.4. Let $\lambda \geq 0$ and let $(u, v, w)$ denote the solution of (1.1). Assume that $(u, v, w)$ does not converge to a solution of the chemotaxis model in the sense of Corollary 1.3. Then

$$
\liminf _{t \rightarrow \infty}\|u(\cdot, t)\|_{L^{1}(\Omega)}=0 .
$$

This result shows that all non-trivial dynamics are covered by the chemotaxis model. It is an open challenge, however, to clarify whether or not in this case we also have $\lim \sup _{t \rightarrow \infty}\|u(\cdot, t)\|_{L^{1}(\Omega)}=0$.

\section{Preliminaries}

Let us first recall that by means of standard arguments involving the contraction mapping principle, one can show that (1.1) possesses a uniquely determined global-in-time classical solution $(u, v, w)$ (cf. Refs. 13-15). Moreover, thanks to the parabolic comparison principle we know that $u$ and $v$ inherit non-negativity from their respective initial data, whereas the inequality $w \geq 0$ immediately results upon an explicit integration of the third equation in (1.1). 
We recall now a well-known property of systems of type (1.1) with a logistic source exhibiting a quadratic decay with respect to $u$ in the first equation.

Lemma 2.1. There exists $C>0$ such that

$$
\int_{\Omega} u(x, t) d x \leq C \quad \text { for all } t \geq 0
$$

and

$$
\int_{t}^{t+1} \int_{\Omega} u^{2}(x, s) d x d s \leq C \quad \text { for all } t \geq 0
$$

Proof. On integration of the first equation in (1.1) we obtain

$$
\begin{aligned}
\frac{d}{d t} \int_{\Omega} u & =r \int_{\Omega} u-\mu \int_{\Omega} u^{2}-\lambda \int_{\Omega} u w \\
& \leq r \int_{\Omega} u-\mu \int_{\Omega} u^{2} \quad \text { for all } t>0
\end{aligned}
$$

because $u$ and $w$ are non-negative. Since $\int_{\Omega} u^{2} \geq \frac{1}{|\Omega|}\left(\int_{\Omega} u\right)^{2}$ by Hölder's inequality, we see that

$$
\frac{d}{d t} \int_{\Omega} u \leq\left(r-\frac{\mu}{|\Omega|} \int_{\Omega} u\right) \cdot \int_{\Omega} u \text { for all } t>0
$$

and thus obtain from an ODE comparison argument that

$$
\int_{\Omega} u \leq \max \left\{\int_{\Omega} u_{0}, \frac{r|\Omega|}{\mu}\right\} \quad \text { for all } t \geq 0
$$

Now integrating (2.3) in time yields

$$
\int_{\Omega} u(x, t+1) d x+\mu \int_{t}^{t+1} \int_{\Omega} u^{2} \leq \int_{\Omega} u(x, t) d x+r \int_{t}^{t+1} \int_{\Omega} u \text { for all } t \geq 0,
$$

which in conjunction with (2.4) proves (2.1) and (2.2).

Secondly, standard smoothing estimates provide the following regularity property of $v$.

Lemma 2.2. For all $q \in[2, \infty)$ there exists $C>0$ such that

$$
\begin{aligned}
& \left\|v_{x}(\cdot, t)\right\|_{L^{q}(\Omega)} \\
& \quad \leq C \cdot\left\{e^{-a t}+\int_{0}^{t}\left[1+(t-s)^{-\alpha}\right] \cdot e^{-a(t-s)} \cdot\|u(\cdot, s)\|_{L^{1}(\Omega)} d s\right\} \quad \text { for all } t>0,
\end{aligned}
$$

where $\alpha:=1-\frac{1}{2 q} \in(0,1)$. 
Proof. Applying the variation-of-constants formula to the second equation in (1.1) and recalling standard smoothing estimates for the Neumann heat semigroup $\left(e^{\tau \Delta}\right)_{\tau \geq 0}$ (see Lemma 1.3 in Ref. 19, for instance), we see that

$$
\begin{aligned}
\left\|v_{x}(\cdot, t)\right\|_{L^{q}(\Omega)}= & \left\|e^{-a t}\left(e^{D_{2} t \Delta} v_{0}\right)_{x}+b \int_{0}^{t} e^{-a(t-s)}\left(e^{D_{2}(t-s) \Delta} u(\cdot, s) d s\right)_{x}\right\|_{L^{q}(\Omega)} \\
\leq & c_{1} e^{-a t}\left\|v_{0 x}\right\|_{L^{q}(\Omega)} \\
& +c_{2} \int_{0}^{t} e^{-a(t-s)}\left(1+(t-s)^{-\frac{1}{2}-\frac{1}{2}\left(1-\frac{1}{q}\right)}\right)\|u(\cdot, s)\|_{L^{1}(\Omega)} d s
\end{aligned}
$$

for all $t \geq 0$ and some $c_{1}>0$ and $c_{2}>0$, which implies (2.5).

Combined with Lemma 2.1, this provides a bound for $v$ in $L^{\infty}\left((0, \infty) ; W^{1, q}(\Omega)\right)$ for arbitrarily large $q$.

Corollary 2.3. For all $q \in[2, \infty)$ we can find $C>0$ fulfilling

$$
\|v(\cdot, t)\|_{W^{1, q}(\Omega)} \leq C \quad \text { for all } t>0 .
$$

Proof. According to Lemma 2.1 there exists $c_{1}>0$ such that

$$
\|u(\cdot, t)\|_{L^{1}(\Omega)} \leq c_{1} \quad \text { for all } t>0 .
$$

Inserted into (2.5), this shows that given $q \in[2, \infty)$, with $\alpha=1-\frac{1}{2 q}$ and some $c_{2}>0$ we have

$$
\begin{aligned}
\left\|v_{x}(\cdot, t)\right\|_{L^{q}(\Omega)} & \leq c_{2} \cdot\left\{e^{-a t}+\int_{0}^{t}\left[1+(t-s)^{-\alpha}\right] \cdot e^{-a(t-s)} \cdot c_{1} d s\right\} \\
& \leq c_{2} \cdot\left\{1+c_{1} \int_{0}^{\infty}\left(1+\sigma^{-\alpha}\right) \cdot e^{-a \sigma} d \sigma\right\} \text { for all } t>0 .
\end{aligned}
$$

Moreover, integrating the second equation in (1.1) in space we see that $(2.7)$ implies boundedness of $v$ in $L^{\infty}\left((0, \infty) ; L^{1}(\Omega)\right)$, which in conjunction with $(2.8)$ proves (2.6).

\section{Boundedness}

\subsection{Pointwise lower bound for $v$}

In order to derive estimates for $w$, in view of the third equation in (1.1) it seems favorable to study lower bounds for $v$. As a preparation, we state a pointwise estimate from below for Neumann heat semigroup $\left(e^{\sigma \Delta}\right)_{\sigma \geq 0}$ in $\Omega$.

Lemma 3.1. Let $D>0$. Then there exists $\Gamma_{0}>0$ such that for all non-negative $z \in C^{0}(\bar{\Omega})$ we have

$$
\left(e^{D \tau \Delta} z\right)(x) \geq \Gamma_{0} \cdot \int_{\Omega} z \quad \text { for all } x \in \Omega \text { and each } \tau \geq 1
$$


Proof. Since for $\tau \geq 1$ we have $e^{D \tau \Delta} z=e^{D(\tau-1) \Delta}\left(e^{D \Delta} z\right)$ and since $e^{D(\tau-1) \Delta}$ is order preserving because of the maximum principle, we may assume that $\tau=1$.

Let us first prove (3.1) for all

$$
\begin{aligned}
z \in S:= & \left\{\varphi \in C_{0}^{\infty}(\mathbb{R}) \mid \varphi \geq 0, \operatorname{supp} \varphi \subset \bar{\Omega}, \text { and there exists } x_{0} \in \Omega\right. \text { such that } \\
& \left.\varphi\left(x_{0}+y\right)=\varphi\left(x_{0}-y\right) \text { and } \varphi_{x}\left(x_{0}+y\right) \leq 0 \text { for all } y>0\right\} .
\end{aligned}
$$

For such $z$, namely, we know that

$$
e^{D t \Delta} z \geq e^{D t \Delta_{C}} z \quad \text { in } \Omega \text { for all } t \geq 0
$$

where $\underline{u}:=e^{D t \Delta_{C}} z$ denotes the solution of the Cauchy problem

$$
\begin{cases}\underline{u}_{t}=D \underline{u}_{x x}, & x \in \mathbb{R}, \quad t>0 \\ \underline{u}(x, 0)=z(x), & x \in \mathbb{R} .\end{cases}
$$

Indeed, since $\underline{u}$ is non-negative and symmetric with respect to some $x_{0} \in \Omega$ and satisfies $\underline{u}_{x} \leq 0$ in $\left(x_{0}, \infty\right) \times(0, \infty)$ by the maximum principle, it follows that $\frac{\partial \underline{u}}{\partial \nu} \leq 0$ on $\partial \Omega \times(0, \infty)$, and hence again by the maximum principle we see that $\underline{u} \leq u:=e^{D t \Delta} z$ in $\Omega \times(0, \infty)$, because $u$ solves the same PDE as $\underline{u}$ but satisfies $\frac{\partial u}{\partial \nu}=0$ on $\partial \Omega \times(0, \infty)$.

Now given $z \in S$, using the explicit representation formula for $e^{D \Delta_{C}} z$ along with the fact that $\operatorname{supp} z \subset \Omega$, we can estimate

$$
\begin{aligned}
\left(e^{D \Delta_{C}} z\right)(x) & =\frac{1}{\sqrt{4 \pi D}} \int_{a}^{b} e^{-\frac{(x-y)^{2}}{4 D}} \cdot z(y) d y \\
& \geq \frac{1}{\sqrt{4 \pi D}} \cdot e^{-\frac{(b-a)^{2}}{4 D}} \cdot \int_{a}^{b} z(y) d y \quad \text { for all } x \in \Omega .
\end{aligned}
$$

In view of $(3.2)$, this proves that for some $\Gamma_{0}>0$ we have

$$
\left(e^{D \Delta} z\right)(x) \geq \Gamma_{0} \cdot \int_{\Omega} z \text { for all } x \in \Omega \text { whenever } z \in S .
$$

However, if $z \in C^{0}(\bar{\Omega})$ is an arbitrary non-trivial non-negative function, then for all $\varepsilon>0$, by a density argument we can pick $N \in \mathbb{N}, c_{1}, \ldots, c_{N}>0$ and $\varphi_{1}, \ldots, \varphi_{N} \in S$ such that

$$
z+\varepsilon \geq \sum_{k=1}^{N} c_{k} \varphi_{k} \geq z-\varepsilon \quad \text { in } \Omega
$$

Thus, by linearity,

$$
e^{D \Delta} z \geq \sum_{k=1}^{N} c_{k} \cdot e^{D \Delta} \varphi_{k}-\varepsilon \geq \Gamma_{0} \cdot \sum_{k=1}^{N} c_{k} \cdot \int_{\Omega} \varphi_{k}-\varepsilon \geq \Gamma_{0} \cdot \int_{\Omega} z-\Gamma_{0} \varepsilon|\Omega|-\varepsilon \quad \text { in } \Omega,
$$

which in the limit $\varepsilon \searrow 0$ yields the desired inequality. 
With the above statement at hand, we can derive a lower bound for $v$ that will be convenient for our purpose.

Lemma 3.2. There exists $\Gamma>0$ such that

$$
\int_{0}^{t} v(x, s) d s \geq \Gamma \cdot \int_{0}^{t-2} \int_{\Omega} u(y, s) d y d s \quad \text { for all } x \in \Omega \text { and } t \geq 2 .
$$

Proof. By means of the variation-of-constants formula, $v$ is represented according to

$$
v(\cdot, t)=e^{-a t} e^{D_{2} t \Delta} v_{0}+b \int_{0}^{t} e^{-a(t-s)} e^{D_{2}(t-s) \Delta} u(\cdot, s) d s \quad \text { for } t \geq 0,
$$

where clearly $e^{-a t} e^{D_{2} t \Delta} v_{0} \geq 0$ in $\Omega$, for $v_{0} \geq 0$. By Lemma 3.1 , for some $\Gamma_{0}>0$ we have

$$
e^{D_{2}(t-s) \Delta} u(\cdot, s) \geq \Gamma_{0} \cdot \int_{\Omega} u(\cdot, s) \quad \text { in } \Omega \text { whenever } t-s \geq 1,
$$

so that for $t \geq 1$ we obtain

$$
\begin{aligned}
v(\cdot, t) & \geq b \int_{0}^{t-1} e^{-a(t-s)} e^{D_{2}(t-s) \Delta} u(\cdot, s) d s \\
& \geq b \Gamma_{0} \cdot \int_{0}^{t-1} e^{-a(t-s)} \cdot \int_{\Omega} u(\cdot, s) d s .
\end{aligned}
$$

Hence an integration in time shows that

$$
\int_{1}^{t} v(x, s) d s \geq b \Gamma_{0} \cdot \int_{1}^{t} \int_{0}^{s-1} e^{-a(s-\sigma)} \cdot \int_{\Omega} u(\cdot, \sigma) d \sigma d s \quad \text { for all } x \in \Omega \text { and } t \geq 1 .
$$

Here, Fubini's theorem yields

$$
\begin{aligned}
b \Gamma_{0} & \cdot \int_{1}^{t} \int_{0}^{s-1} e^{-a(s-\sigma)} \cdot \int_{\Omega} u(\cdot, \sigma) d \sigma d s \\
& =b \Gamma_{0} \cdot \int_{0}^{t-1}\left(\int_{\sigma+1}^{t} e^{-a(s-\sigma)} d s\right) \cdot \int_{\Omega} u(\cdot, \sigma) d \sigma \\
& =\frac{b \Gamma_{0}}{a} \cdot \int_{0}^{t-1}\left(e^{-a}-e^{-a(t-\sigma)}\right) \cdot \int_{\Omega} u(\cdot, \sigma) d \sigma
\end{aligned}
$$

for all $t \geq 1$. Now if $t-\sigma \geq 2$ then $e^{-a}-e^{-a(t-\sigma)} \geq e^{-a}-e^{-2 a}$, so that in the case $t \geq 2$ we can estimate

$$
\begin{aligned}
& \frac{b \Gamma_{0}}{a} \cdot \int_{0}^{t-1}\left(e^{-a}-e^{-a(t-\sigma)}\right) \cdot \int_{\Omega} u(\cdot, \sigma) d \sigma \\
& \quad \geq \frac{b \Gamma_{0}}{a} \cdot\left(e^{-a}-e^{-2 a}\right) \cdot \int_{0}^{t-2} \int_{\Omega} u(x, \sigma) d x d \sigma .
\end{aligned}
$$

Thus, from (3.4)-(3.6) we infer that (3.3) holds if we let $\Gamma:=\frac{b \Gamma_{0}}{a} \cdot\left(e^{-a}-e^{-2 a}\right)$. 
At the cost of a subtractive constant, the upper limit in the time integral on the right of (3.3) can easily be modified conveniently.

Corollary 3.3. With $\Gamma$ as in Lemma 3.2, we can choose $C>0$ such that

$$
\int_{0}^{t} v(x, s) d s \geq \Gamma \cdot \int_{0}^{t} \int_{\Omega} u(y, s) d y d s-C \quad \text { for all } x \in \Omega \text { and } t \geq 0 .
$$

Proof. Since $\int_{\Omega} u(\cdot, t) \leq c_{1}$ for all $t \geq 0$ and some $c_{1}>0$, when $t \geq 2$ using Lemma 3.2 we obtain

$$
\Gamma \int_{0}^{t} \int_{\Omega} u \leq \int_{0}^{t} v(x, s) d s+\Gamma \int_{t-2}^{t} \int_{\Omega} u \leq \int_{0}^{t} v(x, s) d s+2 c_{1} \Gamma \quad \text { for all } x \in \Omega,
$$

whereas in the case $t<2$ we trivially have $\int_{0}^{t} v(x, s) d s \geq 0$ for all $x \in \Omega$ and hence

$$
\Gamma \int_{0}^{t} \int_{\Omega} u \leq c_{1} \Gamma t \leq \int_{0}^{t} v(x, s) d s+2 c_{1} \Gamma \quad \text { for all } x \in \Omega .
$$

This proves the claim upon the choice $C:=2 c_{1} \Gamma$.

\subsection{An estimate for $w$}

A crucial step towards our boundedness proof will be provided by the following lemma.

Lemma 3.4. Let $q \in[2, \infty)$. Then there exists $C>0$ such that

$$
\|w(\cdot, t)\|_{W^{1, q}(\Omega)} \leq C e^{-\rho \Gamma \cdot \int_{0}^{t} \int_{\Omega} u} \cdot\left(1+\int_{0}^{t} \int_{\Omega} u\right) \quad \text { for all } t \geq 0
$$

where $\Gamma$ is as in Corollary 3.3.

Proof. Integrating $w_{t}=-\rho v w$ gives

$$
w(x, t)=w_{0}(x) \cdot e^{-\rho \int_{0}^{t} v(x, s) d s} \quad \text { for } x \in \Omega \text { and } t>0,
$$

so that since Corollary 3.3 implies

$$
e^{-\int_{0}^{t} v(x, s) d s} \leq c_{1} e^{-\Gamma \int_{0}^{t} \int_{\Omega} u(y, s) d y d s} \quad \text { for all } x \in \Omega \text { and } t \geq 0,
$$

with some $c_{1}>0$, we see that

$$
\|w(\cdot, t)\|_{L^{\infty}(\Omega)} \leq c_{1}\left\|w_{0}\right\|_{L^{\infty}(\Omega)} \cdot e^{-\rho \Gamma \int_{0}^{t} \int_{\Omega} u(y, s) d y d s} \quad \text { for all } t \geq 0 .
$$

Moreover, differentiating (3.8) yields

$$
\begin{aligned}
w_{x}(x, t) & =w_{0 x}(x) \cdot e^{-\rho \int_{0}^{t} v(x, s) d s}-\rho w_{0}(x) \cdot e^{-\rho \int_{0}^{t} v(x, s) d s} \cdot \int_{0}^{t} v_{x}(x, s) d s \\
& =: z_{1}(x, t)+z_{2}(x, t) \quad \text { for } x \in \Omega \text { and } t \geq 0 .
\end{aligned}
$$


Here, from (3.9) we obtain

$$
\begin{aligned}
\left|z_{1}(x, t)\right| & \leq\left|w_{0 x}(x)\right| \cdot e^{-\rho \int_{0}^{t} v(x, s) d s} \\
& \leq c_{1}\left|w_{0 x}(x)\right| \cdot e^{-\rho \Gamma \int_{0}^{t} \int_{\Omega} u(y, s) d y d s} \quad \text { for all } x \in \Omega \text { and } t \geq 0,
\end{aligned}
$$

whence

$$
\left\|z_{1}(\cdot, t)\right\|_{L^{q}(\Omega)} \leq c_{1}\left\|w_{0 x}\right\|_{L^{q}(\Omega)} \cdot e^{-\rho \Gamma \int_{0}^{t} \int_{\Omega} u(y, s) d y d s} \quad \text { for all } t \geq 0 .
$$

As to $z_{2}$, we argue by duality and estimate, writing $q^{\prime}:=\frac{q}{q-1}$ and again using (3.9),

$$
\begin{aligned}
\left\|z_{2}(\cdot, t)\right\|_{L^{q}(\Omega)}= & \sup _{\varphi \in C_{0}^{\infty}(\Omega),\|\varphi\|_{L^{q^{\prime}(\Omega)}} \leq 1}\left|\int_{\Omega} z_{2}(x, t) \varphi(x) d x\right| \\
= & \sup _{\varphi \in C_{0}^{\infty}(\Omega),\|\varphi\|_{L^{q^{\prime}(\Omega)}} \leq 1} \mid \rho \int_{\Omega} w_{0}(x) \cdot e^{-\rho \int_{0}^{t} v(x, s) d s} \\
& \cdot \int_{0}^{t} v_{x}(x, s) d s \cdot \varphi(x) d x \mid \\
\leq & c_{1} \rho\left\|w_{0}\right\|_{L^{\infty}(\Omega)} \cdot e^{-\rho \Gamma \int_{0}^{t} \int_{\Omega} u(y, s) d y d s} \\
& \cdot \sup _{\varphi \in C_{0}^{\infty}(\Omega),\|\varphi\|_{L^{q^{\prime}}(\Omega)} \leq 1} \int_{\Omega} \int_{0}^{t}\left|v_{x}(x, s)\right| \cdot|\varphi(x)| d s d x \\
\leq & c_{1} \rho\left\|w_{0}\right\|_{L^{\infty}(\Omega)} \cdot e^{-\rho \Gamma \int_{0}^{t} \int_{\Omega} u(y, s) d y d s} \\
& \cdot \sup _{\varphi \in C_{0}^{\infty}(\Omega),\|\varphi\|_{L^{q^{\prime}}(\Omega)} \leq 1} \int_{0}^{t}\left\|v_{x}(\cdot, s)\right\|_{L^{q}(\Omega)} \cdot\|\varphi\|_{L^{q^{\prime}}(\Omega)} d s \\
\leq & c_{1} \rho\left\|w_{0}\right\|_{L^{\infty}(\Omega)} \cdot e^{-\rho \Gamma \int_{0}^{t} \int_{\Omega} u(y, s) d y d s} \cdot \int_{0}^{t}\left\|v_{x}(\cdot, s)\right\|_{L^{q}(\Omega)} d s
\end{aligned}
$$

for all $t \geq 0$. Now from Lemma 2.2 we know that there exists $c_{2}>0$ such that

$$
\begin{aligned}
& \int_{0}^{t}\left\|v_{x}(\cdot, s)\right\|_{L^{q}(\Omega)} d s \\
& \quad \leq c_{2} \int_{0}^{t}\left\{e^{-a s}+\int_{0}^{s}\left[1+(s-\sigma)^{-\alpha}\right] \cdot e^{-a(s-\sigma)} \cdot\|u(\cdot, \sigma)\|_{L^{1}(\Omega)} d \sigma\right\} d s
\end{aligned}
$$

for all $t \geq 0$, where $\alpha=1-\frac{1}{2 q}$. Since $\int_{0}^{t} e^{-a s} d s \leq \frac{1}{a}$ for all $t \geq 0$, and since Fubini's theorem entails that

$$
\begin{aligned}
\int_{0}^{t} & \int_{0}^{s}\left[1+(s-\sigma)^{-\alpha}\right] \cdot e^{-a(s-\sigma)} \cdot\|u(\cdot, \sigma)\|_{L^{1}(\Omega)} d \sigma d s \\
\quad & \int_{0}^{t}\left(\int_{\sigma}^{t}\left[1+(s-\sigma)^{-\alpha}\right] \cdot e^{-a(s-\sigma)} d s\right) \cdot\|u(\cdot, \sigma)\|_{L^{1}(\Omega)} d \sigma
\end{aligned}
$$




$$
\begin{aligned}
& =\int_{0}^{t}\left(\int_{0}^{t-\sigma}\left[1+\theta^{-\alpha}\right] \cdot e^{-a \theta} d \theta\right) \cdot\|u(\cdot, \sigma)\|_{L^{1}(\Omega)} d \sigma \\
& \leq\left(\int_{0}^{\infty}\left[1+\theta^{-\alpha}\right] \cdot e^{-a \theta} d \theta\right) \cdot \int_{0}^{t}\|u(\cdot, \sigma)\|_{L^{1}(\Omega)} d \sigma \quad \text { for all } t \geq 0,
\end{aligned}
$$

we thus infer that

$$
\int_{0}^{t}\left\|v_{x}(\cdot, s)\right\|_{L^{q}(\Omega)} d s \leq c_{2} \cdot\left\{\frac{1}{a}+\left(\int_{0}^{\infty}\left[1+\theta^{-\alpha}\right] \cdot e^{-a \theta} d \theta\right) \cdot \int_{0}^{t} \int_{\Omega} u(x, \sigma) d x d \sigma\right\}
$$

for all $t \geq 0$. In conjunction with (3.13), (3.11), (3.12) and (3.10), this finally establishes (3.7).

As a consequence of Lemma 3.4, without any further assumption we obtain boundedness of $w$ in $L^{\infty}\left((0, \infty) ; W^{1, q}(\Omega)\right)$ for any finite $q$.

Corollary 3.5. Let $q \in[2, \infty)$. Then there exists $c>0$ such that

$$
\|w(\cdot, t)\|_{W^{1, q}(\Omega)} \leq c \quad \text { for all } t \geq 0 .
$$

Proof. In view of the elementary inequality

$$
z \cdot e^{-\rho \Gamma z} \leq \frac{1}{\rho \Gamma e} \text { for all } z \geq 0,
$$

taking $C$ as provided by Lemma 3.4 , from (3.7) we immediately obtain

$$
\|w(\cdot, t)\|_{W^{1, q}(\Omega)} \leq C \cdot\left(1+\frac{1}{\rho \Gamma e}\right) \quad \text { for all } t \geq 0
$$

and conclude.

\subsection{Proof of Theorem 1.1}

We can now prove our main result on boundedness in (1.1).

Proof of Theorem 1.1. In view of Corollaries 2.3 and 3.5 we only need to show that there exists $C>0$ such that

$$
u(x, t) \leq C \text { for all } x \in \Omega \text { and } t>0 .
$$

To this end, we first observe that according to Lemma 2.1 there exists $c_{1}>0$ such that for each integer $k \geq 2$ we can pick $t_{k} \in(k-2, k-1)$ with the property

$$
\left\|u\left(\cdot, t_{k}\right)\right\|_{L^{2}(\Omega)} \leq c_{1} \quad \text { for all } k \geq 2 .
$$

We now let

$$
M_{k}:=\max _{t \in\left[t_{k}, t_{k}+3\right]}\left(t-t_{k}\right)^{\frac{1}{4}} \cdot\|u(\cdot, t)\|_{L^{\infty}(\Omega)}, \quad k \geq 2
$$


and claim that there exists $c_{2}>0$ satisfying

$$
M_{k} \leq c_{2} \text { for all } k \geq 2 .
$$

For this purpose, we apply the variation-of-constants formula to the first equation in (1.1) to see that

$$
\begin{aligned}
u(\cdot, t)= & e^{D_{1}\left(t-t_{k}\right) \Delta} u\left(\cdot, t_{k}\right)-\chi \int_{t_{k}}^{t} e^{D_{1}(t-s) \Delta}\left(u v_{x}\right)_{x}(\cdot, s) d s \\
& -\xi \int_{t_{k}}^{t} e^{D_{1}(t-s) \Delta}\left(u w_{x}\right)_{x}(\cdot, s) d s \\
& +\int_{t_{k}}^{t} e^{D_{1}(t-s) \Delta} f(u(\cdot, s), w(\cdot, s)) d s \text { for all } t \geq t_{k},
\end{aligned}
$$

where

$$
f(\hat{u}, \hat{w}):=r \hat{u}-\mu \hat{u}^{2}-\lambda \hat{u} \hat{w} \quad \text { for } \hat{u} \geq 0 \text { and } \hat{w} \geq 0 \text {. }
$$

Using well-known smoothing estimates for the Neumann heat semigroup, we find $c_{3}>0$ fulfilling

$$
\begin{aligned}
\left\|e^{D_{1}\left(t-t_{k}\right) \Delta} u\left(\cdot, t_{k}\right)\right\|_{L^{\infty}(\Omega)} & \leq c_{3}\left(t-t_{k}\right)^{-\frac{1}{4}} \cdot\left\|u\left(\cdot, t_{k}\right)\right\|_{L^{2}(\Omega)} \\
& \leq c_{1} c_{3}\left(t-t_{k}\right)^{-\frac{1}{4}} \quad \text { for all } t>t_{k}
\end{aligned}
$$

in view of (3.15). Next, since evidently

$$
f(\hat{u}, \hat{w}) \leq \frac{r^{2}}{4 \mu} \text { for all } \hat{u} \geq 0 \text { and } \hat{w} \geq 0,
$$

the order-preserving property of $\left(e^{\tau \Delta}\right)_{\tau \geq 0}$ implies the one-sided pointwise inequality

$$
\int_{t_{k}}^{t} e^{D_{1}(t-s) \Delta} f(u(\cdot, s) w(\cdot, s)) d s \leq \frac{r^{2}}{2 \mu} \cdot\left(t-t_{k}\right) \quad \text { in } \Omega \text { for all } t>t_{k},
$$

because $e^{\tau \Delta}$ leaves constants unchanged.

In order to estimate the second and the third term on the right of (3.17), we pick any $p \in(1,2)$ and let $q:=\frac{p}{p-1}$ and $\kappa:=\frac{p q+p-q}{q}$. We then have

$$
\kappa=2-\frac{2}{p} \in(0,1)
$$

and $q>p$, and in particular Corollaries 2.3 and 3.5 provide positive constants $c_{4}$ and $c_{5}$ such that

$$
\left\|v_{x}(\cdot, t)\right\|_{L^{q}(\Omega)} \leq c_{4} \quad \text { for all } t \geq 0
$$

and

$$
\left\|w_{x}(\cdot, t)\right\|_{L^{q}(\Omega)} \leq c_{5} \quad \text { for all } t \geq 0
$$


Again according to known smoothing estimates involving a result from Ref. 19, for some $c_{6}>0$ we have

$$
\left\|e^{\tau \Delta} \varphi_{x}\right\|_{L^{\infty}(\Omega)} \leq c_{6} \tau^{-\frac{1}{2}-\frac{1}{2 p}} \cdot\|\varphi\|_{L^{p}(\Omega)} \text { for all } \tau \in\left(0,3 D_{1}\right),
$$

whenever $\varphi \in C^{1}(\bar{\Omega})$. We thereby obtain

$$
\begin{aligned}
\|-\chi & \int_{t_{k}}^{t} e^{D_{1}(t-s) \Delta}\left(u v_{x}\right)_{x}(\cdot, s) d s \|_{L^{\infty}(\Omega)} \\
\leq & c_{6} \chi \cdot D_{1}^{-\frac{1}{2}-\frac{1}{2 p}} \\
& \cdot \int_{t_{k}}^{t}(t-s)^{-\frac{1}{2}-\frac{1}{2 p}}\left\|u(\cdot, s) v_{x}(\cdot, s)\right\|_{L^{p}(\Omega)} d s \quad \text { for all } t \in\left(t_{k}, t_{k}+3\right) .
\end{aligned}
$$

Here, in view of the Hölder inequality we have

$$
\begin{aligned}
\left\|u(\cdot, s) v_{x}(\cdot, s)\right\|_{L^{p}(\Omega)} & \leq\|u(\cdot, s)\|_{L^{\frac{p q}{q-p}}(\Omega)} \cdot\left\|v_{x}(\cdot, s)\right\|_{L^{q}(\Omega)} \\
& \leq\|u(\cdot, s)\|_{L^{\infty}(\Omega)}^{\kappa} \cdot\|u(\cdot, s)\|_{L^{1}(\Omega)}^{1-\kappa} \cdot\left\|v_{x}(\cdot, s)\right\|_{L^{q}(\Omega)} \quad \text { for all } s \geq 0
\end{aligned}
$$

so that using (3.21) and the boundedness of $u$ in $L^{\infty}\left((0, \infty) ; L^{1}(\Omega)\right)$ asserted by Lemma 2.1, we see that

$$
\begin{aligned}
\left\|u(\cdot, s) v_{x}(\cdot, s)\right\|_{L^{p}(\Omega)} & \leq c_{7}\|u(\cdot, s)\|_{L^{\infty}(\Omega)}^{\kappa} \\
& \leq c_{7} \cdot\left(s-t_{k}\right)^{-\frac{\kappa}{4}} \cdot M_{k}^{\kappa} \quad \text { for all } s \in\left(t_{k}, t_{k}+3\right),
\end{aligned}
$$

with some $c_{7}>0$. Inserted into (3.24), this yields $c_{8}>0$ such that

$$
\begin{aligned}
\left\|-\chi \int_{t_{k}}^{t} e^{D_{1}(t-s) \Delta}\left(u v_{x}\right)_{x}(\cdot, s) d s\right\|_{L^{\infty}(\Omega)} & \leq c_{8} M_{k}^{\kappa} \cdot \int_{t_{k}}^{t}(t-s)^{-\frac{1}{2}-\frac{1}{2 p}} \cdot\left(s-t_{k}\right)^{-\frac{\kappa}{4}} d s \\
& =c_{8} M_{k}^{\kappa} \cdot \int_{0}^{1}(1-\sigma)^{-\frac{1}{2}-\frac{1}{2 p}} \cdot \sigma^{-\frac{\kappa}{4}} d \sigma \\
& =: c_{9} M_{k}^{\kappa} \quad \text { for all } t \in\left(t_{k}, t_{k}+3\right),
\end{aligned}
$$

because $\frac{1}{2}+\frac{1}{2 p}+\frac{\kappa}{4}=1$ by $(3.20)$.

Quite a similar reasoning based on (3.22) shows that there exists $c_{10}>0$ such that

$$
\left\|-\xi \int_{t_{k}}^{t} e^{D_{1}(t-s) \Delta}\left(u w_{x}\right)_{x}(\cdot, s) d s\right\|_{L^{\infty}(\Omega)} \leq c_{10} M_{k}^{\kappa} \quad \text { for all } t \in\left(t_{k}, t_{k}+3\right) .
$$

We now collect (3.18), (3.19), (3.25) and (3.26) to obtain from (3.17) and the nonnegativity of $u$ that

$$
\left(t-t_{k}\right)^{\frac{1}{4}} \cdot\|u(\cdot, t)\|_{L^{\infty}(\Omega)} \leq c_{11}+c_{12} M_{k}^{\kappa} \quad \text { for all } t \in\left(t_{k}, t_{k}+3\right),
$$


with some $c_{11}>0$ and $c_{12}>0$. Since $\kappa \in(0,1)$, we can employ Young's inequality here to derive from this the existence of $c_{13}>0$ fulfilling

$$
\left(t-t_{k}\right)^{\frac{1}{4}} \cdot\|u(\cdot, t)\|_{L^{\infty}(\Omega)} \leq \frac{1}{2} M_{k}+c_{13} \quad \text { for all } t \in\left(t_{k}, t_{k}+3\right) .
$$

This entails

$$
M_{k} \leq \frac{1}{2} M_{k}+c_{13}
$$

and hence

$$
M_{k} \leq 2 c_{13} \text { for all } k \geq 2 \text {. }
$$

This proves that

$$
\|u(\cdot, t)\|_{L^{\infty}(\Omega)} \leq 2 c_{13} \quad \text { for all } t \in\left(t_{k}+1, t_{k}+3\right) \text { and each } k \geq 2,
$$

as the fact that $t_{k} \in(k-2, k-1)$ implies that $\left(t_{k}+1, t_{k}+3\right) \supset[k, k+1]$ for all $k \geq 2$, we conclude that

$$
\|u(\cdot, t)\|_{L^{\infty}(\Omega)} \leq 2 c_{13} \text { for all } t \geq 2 .
$$

In view of the evident boundedness of $u$ in $\Omega \times(0,2)$, this leads to (3.14) and thus completes the proof.

\section{Decay of $w$ and Asymptotic Behavior}

Our next goal is to prove that under the smallness assumption $(1.3), w(\cdot, t)$ decays exponentially as $t \rightarrow \infty$, where in view of our final purpose (cf. Corollary 4.4) it seems favorable to make sure that this convergence takes place at least in $W^{1, q}(\Omega)$ for some $q>1$. In fact, we shall assert a result of this type in the space $W^{1, q}(\Omega)$ for any $q<\infty$.

The following preparation for this results from a straightforward combination of Ehrling's lemma with the Poincaré inequality, whence its proof may be omitted here.

Lemma 4.1. Let $p \in(2, \infty)$. Then for all $\varepsilon>0$ there exists $K(\varepsilon)>0$ such that

$$
\|z\|_{L^{p}(\Omega)} \leq \varepsilon\left\|z_{x}\right\|_{L^{2}(\Omega)}^{2}+K(\varepsilon) \cdot\|z\|_{L^{2}(\Omega)}^{2} \quad \text { for all } z \in W^{1,2}(\Omega) .
$$

In view of Lemma 3.4, as our only task towards the desired decay result, it remains to ensure that $\int_{0}^{t} \int_{\Omega} u$ becomes conveniently large for large $t$. We note that without any proliferation terms in the first equation in (1.1), that is, under the assumption $r=\mu=\lambda=0$, simply integrating this equation in space would yield $\int_{\Omega} u(\cdot, t) \equiv \int_{\Omega} u_{0}$, which for non-trivial $u_{0}$ would imply $\int_{0}^{t} \int_{\Omega} u \rightarrow \infty$ as $t \rightarrow \infty$. However, in the present setting we suspect that the evolution of the total mass might be more involved, and accordingly it does not seem trivial to exclude the possibility that $\int_{\Omega} u(\cdot, t)$ might even decay as $t \rightarrow \infty$. However, we can rule out the latter type of behavior under the additional assumption that $\lambda w \leq r$ in $\Omega$. We shall see in Proposition 5.1 below that this cannot be relaxed. 
Our technique to derive this lower bound appears to be new in this context: In order to obtain a positive lower bound for $\int_{\Omega} u(\cdot, t)$ for all $t>0$, we deduce a differential inequality for the seminorm of $u(\cdot, t)$ in $L^{\gamma}(\Omega)$ for some positive $\gamma$ which is smaller than one. Unlike the case when $\gamma>1$, in a corresponding testing procedure the term stemming from self-diffusion now has a favorable sign in respect of preventing decay (cf. (4.6) below).

Lemma 4.2. Suppose that $u_{0} \not \equiv 0$, that $\lambda \geq 0$, and that

$$
\lambda \cdot\left\|w_{0}\right\|_{L^{\infty}(\Omega)} \leq r
$$

Then there exist $\gamma \in(0,1)$ and $c>0$ such that

$$
\int_{\Omega} u^{\gamma}(x, t) d x \geq c \quad \text { for all } t \in[0, \infty)
$$

Proof. Since $v>0$ in $\bar{\Omega} \times(0, \infty)$ by the strong maximum principle, upon a small time shift if necessary we may assume that

$$
\eta:=r-\lambda \cdot\left\|w_{0}\right\|_{L^{\infty}(\Omega)}>0
$$

Let us fix any $q \in(2, \infty)$ and then apply Corollaries 2.3 and 3.5 to obtain constants $c_{1}>0$ and $c_{2}>0$ such that

$$
\left\|v_{x}(\cdot, t)\right\|_{L^{q}(\Omega)} \leq c_{1} \quad \text { for all } t>0
$$

and

$$
\left\|w_{x}(\cdot, t)\right\|_{L^{q}(\Omega)} \leq c_{2} \quad \text { for all } t>0
$$

With $K(\cdot)$ as provided by Lemma 4.1, we now pick $\gamma \in(0,1)$ sufficiently close to 1 fulfilling

$$
\left\{\chi^{2} c_{1}^{2} \cdot K\left(\frac{D_{1}^{2}}{4 \chi^{2} c_{1}^{2}}\right)+\xi^{2} c_{2}^{2} \cdot K\left(\frac{D_{1}^{2}}{4 \xi^{2} c_{2}^{2}}\right)\right\} \cdot \frac{2(1-\gamma)}{D_{1}}<\frac{\eta}{2} .
$$

Testing the first equation in (1.1) by $u^{\gamma-1}$ we then obtain

$$
\begin{aligned}
\frac{1}{\gamma} \frac{d}{d t} \int_{\Omega} u^{\gamma}= & D_{1}(1-\gamma) \int_{\Omega} u^{\gamma-2} u_{x}^{2}-\chi(1-\gamma) \int_{\Omega} u^{\gamma-1} u_{x} v_{x}-\xi(1-\gamma) \int_{\Omega} u^{\gamma-1} u_{x} w_{x} \\
& +r \int_{\Omega} u^{\gamma}-\mu \int_{\Omega} u^{\gamma+1}-\lambda \int_{\Omega} u^{\gamma} w \quad \text { for all } t>0
\end{aligned}
$$

Here, Young's inequality gives

$$
\left|-\chi(1-\gamma) \int_{\Omega} u^{\gamma-1} u_{x} v_{x}\right| \leq \frac{D_{1}(1-\gamma)}{8} \cdot \int_{\Omega} u^{\gamma-2} u_{x}^{2}+\frac{2 \chi^{2}(1-\gamma)}{D_{1}} \cdot \int_{\Omega} u^{\gamma} v_{x}^{2},
$$


and Hölder's inequality along with (4.3) yields

$$
\begin{aligned}
\frac{2 \chi^{2}(1-\gamma)}{D_{1}} \cdot \int_{\Omega} u^{\gamma} v_{x}^{2} & \leq \frac{2 \chi^{2}(1-\gamma)}{D_{1}} \cdot\left(\int_{\Omega} u^{\frac{q \gamma}{q-2}}\right)^{\frac{q-2}{q}} \cdot\left(\int_{\Omega}\left|v_{x}\right|^{q}\right)^{\frac{2}{q}} \\
& \leq \frac{2 \chi^{2} c_{1}^{2}(1-\gamma)}{D_{1}} \cdot\left(\int_{\Omega} u^{\frac{q \gamma}{q-2}}\right)^{\frac{q-2}{q}} .
\end{aligned}
$$

We apply Lemma 4.1 to $z:=u^{\frac{\gamma}{2}}$ to estimate

$$
\begin{aligned}
\left(\int_{\Omega} u^{\frac{q \gamma}{q-2}}\right)^{\frac{q-2}{q}} & =\left\|u^{\frac{\gamma}{2}}\right\|_{L^{\frac{2 q}{q-2}}(\Omega)}^{2} \\
& \leq \frac{D_{1}^{2}}{4 \chi^{2} c_{1}^{2}}\left\|\left(u^{\frac{\gamma}{2}}\right)_{x}\right\|_{L^{2}(\Omega)}^{2}+K\left(\frac{D_{1}^{2}}{4 \chi^{2} c_{1}^{2}}\right) \cdot\left\|u^{\frac{\gamma}{2}}\right\|_{L^{2}(\Omega)}^{2} \\
& =\frac{D_{1}^{2} \gamma^{2}}{16 \chi^{2} c_{1}^{2}} \cdot \int_{\Omega} u^{\gamma-2} u_{x}^{2}+K\left(\frac{D_{1}^{2}}{4 \chi^{2} c_{1}^{2}}\right) \cdot \int_{\Omega} u^{\gamma}
\end{aligned}
$$

and thus infer from (4.7) and (4.8) that

$$
\begin{aligned}
\left|-\chi(1-\gamma) \int_{\Omega} u^{\gamma-1} u_{x} v_{x}\right| \leq & \frac{D_{1}(1-\gamma)}{8} \cdot\left(1+\gamma^{2}\right) \cdot \int_{\Omega} u^{\gamma-2} u_{x}^{2} \\
& +\frac{2 \chi^{2} c_{1}^{2}(1-\gamma)}{D_{1}} \cdot K\left(\frac{D_{1}^{2}}{4 \chi^{2} c_{1}^{2}}\right) \cdot \int_{\Omega} u^{\gamma} .
\end{aligned}
$$

By the same arguments relying on (4.4) rather than on (4.3), we see that

$$
\begin{aligned}
\left|-\xi(1-\gamma) \int_{\Omega} u^{\gamma-1} u_{x} w_{x}\right| \leq & \frac{D_{1}(1-\gamma)}{8} \cdot\left(1+\gamma^{2}\right) \cdot \int_{\Omega} u^{\gamma-2} u_{x}^{2} \\
& +\frac{2 \xi^{2} c_{2}^{2}(1-\gamma)}{D_{1}} \cdot K\left(\frac{D_{1}^{2}}{4 \xi^{2} c_{2}^{2}}\right) \cdot \int_{\Omega} u^{\gamma}
\end{aligned}
$$

Next, in order to estimate the second last term in (4.6) we invoke the GagliardoNirenberg inequality to find some $c_{3}>0$ such that

$$
\begin{aligned}
& \mu \int_{\Omega} u^{\gamma+1}=\mu\left\|u^{\frac{\gamma}{2}}\right\|_{L^{\frac{2(\gamma+1)}{\gamma}}}^{\frac{2(\gamma+1)}{\gamma}(\Omega)} \\
& \leq c_{3} \cdot\left(\left\|\left(u^{\frac{\gamma}{2}}\right)_{x}\right\|_{L^{2}(\Omega)}^{\frac{2(\gamma+1)}{\gamma}} \cdot d x \cdot\left\|u^{\frac{\gamma}{2}}\right\|_{L^{2}(\Omega)}^{\frac{2(\gamma+1)}{\gamma} \cdot(1-d)}+\left\|u^{\frac{\gamma}{2}}\right\|_{L^{2}(\Omega)}^{\frac{2(\gamma+1)}{\gamma}}\right),
\end{aligned}
$$

where

$$
-\frac{\gamma}{2(\gamma+1)}=\left(1-\frac{1}{2}\right) d-\frac{1}{2}(1-d)=d-\frac{1}{2},
$$

that is,

$$
d=\frac{1}{2(\gamma+1)}
$$


Therefore,

$$
\mu \int_{\Omega} u^{\gamma+1} \leq c_{3} \cdot\left(\left\|\left(u^{\frac{\gamma}{2}}\right)_{x}\right\|_{L^{2}(\Omega)}^{\frac{1}{\gamma}} \cdot\left\|u^{\frac{\gamma}{2}}\right\|_{L^{2}(\Omega)}^{\frac{2 \gamma+1}{\gamma}}+\left\|u^{\frac{\gamma}{2}}\right\|_{L^{2}(\Omega)}^{\frac{2(\gamma+1)}{\gamma}}\right)
$$

so that an application of Young's inequality yields $c_{4}>0$ such that

$$
\begin{aligned}
\mu \int_{\Omega} u^{\gamma+1} & \leq \frac{D_{1}(1-\gamma)}{2} \cdot \int_{\Omega} u^{\gamma-2} u_{x}^{2}+c_{4} \cdot\left\|u^{\frac{\gamma}{2}}\right\|_{L^{2}(\Omega)}^{\frac{2(2 \gamma+1)}{2 \gamma-1}}+c_{3} \cdot\left\|u^{\frac{\gamma}{2}}\right\|_{L^{2}(\Omega)}^{\frac{2(\gamma+1)}{\gamma}} \\
& =\frac{D_{1}(1-\gamma)}{2} \cdot \int_{\Omega} u^{\gamma-2} u_{x}^{2}+c_{4} \cdot\left(\int_{\Omega} u^{\gamma}\right)^{\frac{2 \gamma+1}{2 \gamma-1}}+c_{3} \cdot\left(\int_{\Omega} u^{\gamma}\right)^{\frac{\gamma+1}{\gamma}} .
\end{aligned}
$$

Finally, using that $w_{t} \leq 0$, from (4.2) we gain the inequality

$$
\lambda \int_{\Omega} u^{\gamma} w \leq \lambda \int_{\Omega} u^{\gamma} w_{0} \leq(r-\eta) \cdot \int_{\Omega} u^{\gamma},
$$

whence collecting (4.6), (4.9), (4.10), (4.11) and (4.12) we obtain

$$
\begin{aligned}
\frac{1}{\gamma} \frac{d}{d t} \int_{\Omega} u^{\gamma} \geq & \left\{D_{1}(1-\gamma)-\frac{D_{1}(1-\gamma)}{8} \cdot\left(1+\gamma^{2}\right)-\frac{D_{1}(1-\gamma)}{8} \cdot\left(1+\gamma^{2}\right)\right. \\
& \left.-\frac{D_{1}(1-\gamma)}{2}\right\} \cdot \int_{\Omega} u^{\gamma-2} u_{x}^{2}+\left\{r-\frac{2 \chi^{2} c_{1}^{2}(1-\gamma)}{D_{1}} \cdot K\left(\frac{D_{1}^{2}}{4 \chi^{2} c_{1}^{2}}\right)\right. \\
& \left.-\frac{2 \xi^{2} c_{2}^{2}(1-\gamma)}{D_{1}} \cdot K\left(\frac{D_{1}^{2}}{4 \xi^{2} c_{2}^{2}}\right)-(r-\eta)\right\} \cdot \int_{\Omega} u^{\gamma} \\
& -c_{4} \cdot\left(\int_{\Omega} u^{\gamma}\right)^{\frac{2 \gamma+1}{2 \gamma-1}}-c_{3} \cdot\left(\int_{\Omega} u^{\gamma}\right)^{\frac{\gamma+1}{\gamma}} \text { for all } t>0 .
\end{aligned}
$$

In view of (4.5) and the fact that $\gamma<1$, this shows that $y(t):=\int_{\Omega} u^{\gamma}(x, t)$ satisfies

$$
\frac{1}{\gamma} y^{\prime}(t) \geq \frac{\eta}{2} y(t)-c_{4} y^{\kappa_{1}}(t)-c_{3} y^{\kappa_{2}}(t) \text { for all } t>0
$$

where $\kappa_{1}:=\frac{2 \gamma+1}{2 \gamma-1}$ and $\kappa_{2}:=\frac{\gamma+1}{\gamma}$. Since both $\kappa_{1}>1$ and $\kappa_{2}>1$, an ODE comparison shows that

$$
y(t) \geq c_{5}:=\min \left\{y(0), y_{s}\right\} \text { for all } t>0,
$$

where $y_{s}$ is the unique positive solution of $\frac{\eta}{2} y_{s}-c_{4} y_{s}^{\kappa_{1}}-c_{3} y_{s}^{\kappa_{2}}=0$. Since $c_{5}$ is positive in view of the fact that $u_{0} \not \equiv 0$, the proof is complete.

On an application of Hölder's inequality, from Lemma 4.2 we immediately obtain the following. 
Corollary 4.3. Let $\lambda \geq 0$, and assume that $u_{0} \not \equiv 0$ and

$$
\lambda\left\|w_{0}\right\|_{L^{\infty}(\Omega)} \leq r .
$$

Then there exists $c>0$ such that

$$
\int_{\Omega} u(x, t) d x \geq c \quad \text { for all } t \in[0, \infty) .
$$

Proof. According to Lemma 4.2, let us choose $\gamma \in(0,1)$ and $c_{1}>0$ in such a way that $\int_{\Omega} u^{\gamma}(x, t) d x \geq c_{1}$ for all $t \geq 0$. Since $\gamma<1$, the Hölder inequality then says that $\int_{\Omega} u^{\gamma} \leq|\Omega|^{1-\gamma}\left(\int_{\Omega} u\right)^{\gamma}$, whereupon we infer that (4.13) holds if we let $c:=c_{1}^{\frac{1}{\gamma}}|\Omega|^{1-\frac{1}{\gamma}}$, for instance.

\subsection{Proof of Theorem 1.2}

We are now ready to establish our main result on decay of the ECM.

Proof of Theorem 1.2. Given $q \in[2, \infty)$, Lemma 3.4 yields $c_{1}>0$ and $\Gamma>0$ such that

$$
\|w(\cdot, t)\|_{W^{1, q}(\Omega)} \leq c_{1} e^{-\Gamma \int_{0}^{t} \int_{\Omega} u} \cdot\left(1+\int_{0}^{t} \int_{\Omega} u\right) \quad \text { for all } t \geq 0,
$$

which implies that for some $c_{2}>0$ we have

$$
\|w(\cdot, t)\|_{W^{1, q}(\Omega)} \leq c_{2} e^{-\frac{\Gamma}{2} \int_{0}^{t} \int_{\Omega} u} \text { for all } t \geq 0,
$$

because $(1+z) e^{-\Gamma z} \leq\left(1+\frac{2}{\Gamma e}\right) \cdot e^{-\frac{\Gamma}{2} z}$ for all $z \geq 0$. With $c>0$ as given by Corollary 4.3 , we therefore obtain

$$
\|w(\cdot, t)\|_{W^{1, q}(\Omega)} \leq c_{2} e^{-\frac{\Gamma}{2} c t} \quad \text { for all } t \geq 0
$$

which proves the assertion.

\subsection{Proof of Corollary 1.3 and Theorem 1.4}

The proof of Corollary 1.3 will result directly from the following.

Lemma 4.4. Let $\lambda \geq 0$ and $u_{0} \not \equiv 0$, and suppose that (1.3) holds. Then there exist $C>0$ and $\theta>0$ such that

$$
\begin{gathered}
\sup _{t>t_{0}} \| u(\cdot, t)-e^{D_{1}\left(t-t_{0}\right) \Delta} u_{0}-\int_{t_{0}}^{t} e^{D_{1}(t-s) \Delta}\left\{\chi\left(u v_{x}\right)_{x}(\cdot, s)+r u(\cdot, s)\right. \\
\left.-\mu u^{2}(\cdot, s)\right\} d s \|_{L^{\infty}(\Omega)} \leq C e^{-\theta t_{0}} \quad \text { for all } t_{0} \geq 0 .
\end{gathered}
$$

Proof. According to Theorems 1.1 and 1.2, there exist $c_{1}>0$ and $\theta>0$ such that $\|u(\cdot, t)\|_{L^{\infty}(\Omega)} \leq c_{1} \quad$ and $\quad\|w(\cdot, t)\|_{L^{\infty}(\Omega)}+\left\|w_{x}(\cdot, t)\right\|_{L^{2}(\Omega)} \leq c_{1} e^{-\theta t} \quad$ for all $t>0$. 
Now by the variation-of-constants formula applied to the first equation in (1.1), for all $t_{0} \geq 0$ and each $t>t_{0}$ we have

$$
\begin{aligned}
& u(\cdot, t)-e^{D_{1}\left(t-t_{0}\right) \Delta} u_{0}-\int_{t_{0}}^{t} e^{D_{1}(t-s) \Delta}\left\{-\chi\left(u(\cdot, s) v_{x}(\cdot, s)\right)_{x}+r u(\cdot, s)-\mu u^{2}(\cdot, s)\right\} d s \\
& =-\xi \int_{t_{0}}^{t} e^{D_{1}(t-s) \Delta}\left(u(\cdot, s) w_{x}(\cdot, s)\right)_{x} d s-\lambda \int_{t_{0}}^{t} e^{D_{1}(t-s) \Delta} u(\cdot, s) w(\cdot, s) d s \\
& =: I_{1}(t)+I_{2}(t) .
\end{aligned}
$$

Here, the smoothing action of the heat semigroup (cf. (3.23)) along with (4.16) ensures that for some $c_{2}>0$ the inequality

$$
\begin{aligned}
\left\|I_{1}(t)\right\|_{L^{\infty}(\Omega)} & \leq c_{2} \int_{t_{0}}^{t}\left(1+(t-s)^{-\frac{3}{4}}\right)\left\|u(\cdot, s) w_{x}(\cdot, s)\right\|_{L^{2}(\Omega)} d s \\
& \leq c_{1}^{2} c_{2} \int_{t_{0}}^{t}\left(1+(t-s)^{-\frac{3}{4}}\right) e^{-\theta s} d s
\end{aligned}
$$

is valid for all $t>t_{0}$. Thus, for large $t$ we have

$$
\begin{aligned}
\left\|I_{1}(t)\right\|_{L^{\infty}(\Omega)} & \leq c_{1}^{2} c_{2} \cdot\left\{2 \int_{t_{0}}^{t-1} e^{-\theta s} d s+2 e^{-\theta t_{0}} \int_{t-1}^{t}(t-s)^{-\frac{3}{4}} d s\right\} \\
& \leq c_{1}^{2} c_{2} \cdot\left\{\frac{2}{\theta} e^{-\theta t_{0}}+8 e^{-\theta t_{0}}\right\} \quad \text { for all } t \geq t_{0}+1,
\end{aligned}
$$

whereas for $t$ close to $t_{0}$ we can estimate

$$
\begin{aligned}
\left\|I_{1}(t)\right\|_{L^{\infty}(\Omega)} & \leq c_{1}^{2} c_{2} e^{-\theta t_{0}} \cdot \int_{t_{0}}^{t}\left(1+(t-s)^{-\frac{3}{4}}\right) d s \\
& =c_{1}^{2} c_{2} e^{-\theta t_{0}} \cdot\left(t-t_{0}+4\left(t-t_{0}\right)^{\frac{1}{4}}\right) \\
& \leq 5 c_{1}^{2} c_{2} e^{-\theta t_{0}} \quad \text { for all } t \in\left(t_{0}, t_{0}+1\right) .
\end{aligned}
$$

Moreover, by the maximum principle and (4.16),

$$
\begin{aligned}
\left\|I_{2}(t)\right\|_{L^{\infty}(\Omega)} & \leq \lambda \int_{t_{0}}^{t}\|u(\cdot, s) w(\cdot, s)\|_{L^{\infty}(\Omega)} d s \\
& \leq \lambda c_{1}^{2} \int_{t_{0}}^{t} e^{-\theta s} d s \\
& \leq \lambda c_{1}^{2} \frac{1}{\theta} e^{-\theta t_{0}} \quad \text { for all } t>t_{0} .
\end{aligned}
$$


Combining (4.17)-(4.21), we immediately arrive at

$$
\begin{gathered}
\sup _{t>t_{0}} \| u(\cdot, t)-e^{D_{1}\left(t-t_{0}\right) \Delta} u_{0}-\int_{t_{0}}^{t} e^{D_{1}(t-s) \Delta}\left\{\chi\left(u v_{x}\right)_{x}(\cdot, s)+r u(\cdot, s)\right. \\
\left.-\mu u^{2}(\cdot, s)\right\} d s \|_{L^{\infty}(\Omega)} \leq C e^{-\theta t_{0}} \quad \text { for all } t_{0} \geq 0 .
\end{gathered}
$$

Proof of Corollary 1.3. The estimate for $u$ is an immediate consequence of Lemma 4.4. The statement concerning $v$ is evident, since the equations for $v$ in the cancer invasion model (1.1) and in the chemotaxis model (1.2) are identical, hence they have the same solution operator $\Phi_{2}$.

Proof of Theorem 1.4. Let us assume that $(u, v, w)$ does not behave as stated in Corollary 1.3 but

$$
\liminf _{t \rightarrow \infty}\|u(\cdot, t)\|_{L^{1}(\Omega)}>0
$$

Then the above arguments, starting from (4.14), apply to guarantee that the conclusion of Theorem 1.2 is still valid. We therefore can fully carry over the proof of Lemma 4.4 and hence of Corollary 1.3 to infer that $(u, v, w)$ satisfies $(1.4)$ and (1.5), contrary to the hypothesis.

\section{Linearization and Sharpness of the Condition for Decay}

\subsection{Linear analysis}

In this section we perform a linear analysis of model (1.1). We will show that assumption (1.3) is necessary for the tumor to invade, which, of course, is the interesting case.

The spatially homogeneous steady states for model (1.1) are

$$
P_{1}(0,0,0), \quad P_{2}\left(\frac{r}{\mu}, \frac{b r}{a \mu}, 0\right), \quad P_{3}\left(0,0, w^{*}\right), \quad w^{*}>0,
$$

where $P_{3}$ denotes a continuum of homogeneous steady states.

We assume that $(\bar{u}, \bar{v}, \bar{w})$ is a given homogeneous steady state, then the linearization of $(1.1)$ in $(\bar{u}, \bar{v}, \bar{w})$ is given by

$$
\begin{aligned}
u_{t} & =D_{1} u_{x x}-\chi \bar{u} v_{x x}-\xi \bar{u} w_{x x}+r u-2 \mu \bar{u} u-\lambda \bar{u} w-\lambda u \bar{w}, \\
v_{t} & =D_{2} v_{x x}-a v+b u \\
w_{t} & =-\rho \bar{v} w-\rho v \bar{w} .
\end{aligned}
$$

Our spatial domain $\Omega$ is an open, bounded interval in $\mathbb{R}^{n}$. Hence there exist a family of eigenvalues and eigenfunctions of the Laplacian on $\Omega$ equipped with the appropriate boundary conditions. The eigenvalues are countable and we denote 
them by $-\nu_{k}$, where $\nu_{k} \geq 0$. The corresponding eigenfunctions are denoted by $\varphi_{k}(x)$.

If we linearize in $P_{1}$ and apply Fourier transform then we obtain the following Jacobians: At $P_{1}(0,0,0)$ we have

$$
J(0,0,0)=\left(\begin{array}{ccc}
-\nu_{k} D_{1}+r & 0 & 0 \\
b & -\nu_{k} D_{2}-a & 0 \\
0 & 0 & 0
\end{array}\right),
$$

hence the eigenvalues are $\lambda_{1}=-\nu_{k} D_{1}+r, \lambda_{2}=0$ and $\lambda_{3}=-\nu_{k} D_{2}-a$. If, for example, we study a domain with homogeneous Neumann boundary conditions (or a periodic domain), then $\nu_{1}=0$ is the leading eigenvalue of the Laplacian and the steady state $P_{1}$ has an unstable eigenvalue $\lambda_{1}=r>0$; that is, a tumor is starting to grow. The eigenvalue $\lambda_{2}=0$ is an indication of the continuum of steady states expressed in $P_{3}$.

If we linearize in $P_{3}\left(0,0, w^{*}\right)$ we obtain a Jacobian of

$$
J\left(0,0, w^{*}\right)=\left(\begin{array}{ccc}
-\nu_{k} D_{1}+r-\lambda w^{*} & 0 & 0 \\
b & -\nu_{k} D_{2}-a & 0 \\
0 & -\rho w^{*} & 0
\end{array}\right)
$$

and the eigenvalues are $\lambda_{1}=-\nu_{k} D_{1}+r-\lambda w^{*}, \lambda_{2}=0$ and $\lambda_{3}=-\nu_{k} D_{2}-a$. For the tumor to be able to invade the healthy tissue, we need $\lambda_{1}>0$ at least for small $k$. This gives a necessary condition for tumor invasion of

$$
r>\lambda w^{*} .
$$

Without this condition, the tumor would not invade the healthy tissue.

\subsection{A counterexample involving large $w_{0}$}

By using flat solutions as counterexamples, we can show that the smallness condition (1.3) in fact cannot be relaxed.

Proposition 5.1. Suppose that $\lambda>0$. Then for all $\varepsilon>0$ there exist positive smooth functions $u_{0}, v_{0}$ and $w_{0}$ such that

$$
\lambda\left\|w_{0}\right\|_{L^{\infty}(\Omega)} \leq r+\varepsilon,
$$

but such that the corresponding solution $(u, v, w)$ of (1.1) satisfies

$$
w(x, t)>\frac{r}{\lambda} \text { for all } x \in \Omega \text { and } t>0 .
$$

In particular, $u_{0}, v_{0}$ and $w_{0}$ can be chosen to be spatially constant.

Proof. We fix $\alpha \in(0, a)$ such that $2 \alpha \leq \varepsilon$, and let

$$
w_{0}(x):=\frac{r+2 \alpha}{\lambda}, \quad x \in \bar{\Omega} .
$$


It is then possible to choose $\delta>0$ small enough such that

$$
w_{0} \cdot e^{-\rho \cdot\left(1+\frac{b}{a-\alpha}\right) \cdot \frac{\delta}{\alpha}} \geq \frac{r+\alpha}{\lambda} \quad \text { in } \bar{\Omega},
$$

and pick any constant functions $u_{0}$ and $v_{0}$ satisfying

$$
0<u_{0}<\delta \text { and } 0<v_{0}<\delta \text { in } \bar{\Omega} \text {. }
$$

We now let $(u, v, w)$ denote the solution of (1.1) emanating from $\left(u_{0}, v_{0}, w_{0}\right)$ and note that clearly $(u, v, w) \equiv(u(t), v(t), w(t))$ is spatially constant for all times and thus actually satisfies the ODE system

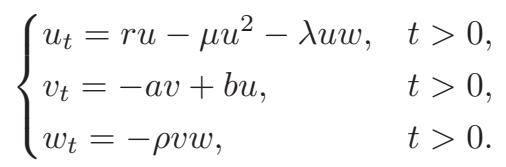

By (5.7), the number

$$
T:=\sup \left\{\tilde{T}>0 \mid u(t) \leq \delta e^{-\alpha t} \text { for all } t \in[0, \tilde{T})\right\} \leq \infty
$$

is well-defined and positive. According to (5.7) and the second equation in (5.8), for $t \in(0, T)$ we have

$$
\begin{aligned}
v(t) & =v_{0} e^{-a t}+b \int_{0}^{t} e^{-a(t-s)} u(s) d s \\
& \leq \delta e^{-a t}+b \delta \int_{0}^{t} e^{-a(t-s)} e^{-\alpha s} d s \\
& =\delta e^{-a t}+\frac{b \delta}{a-\alpha}\left(e^{-\alpha t}-e^{-a t}\right) \\
& \leq \delta e^{-a t}+\frac{b \delta}{a-\alpha} e^{-\alpha t} \\
& \leq\left(1+\frac{b}{a-\alpha}\right) \cdot \delta e^{-\alpha t} \quad \text { for all } t \in(0, T),
\end{aligned}
$$

because $\alpha<a$. Now the third equation in (5.8) shows that

$$
\begin{aligned}
w(t) & =w_{0} \cdot e^{-\rho \int_{0}^{t} v(s) d s} \\
& \geq w_{0} \cdot e^{-\rho\left(1+\frac{b}{a-\alpha}\right) \delta \cdot \int_{0}^{t} e^{-\alpha s}} d s \\
& \geq w_{0} \cdot e^{-\rho\left(1+\frac{b}{a-\alpha}\right) \cdot \frac{\delta}{\alpha}} \quad \text { for all } t \in(0, T),
\end{aligned}
$$

which in view of (5.6) yields

$$
w(t) \geq \frac{r+\alpha}{\lambda} \text { for all } t \in(0, T)
$$

Inserted into the first equation in (5.8), this implies that

$$
\begin{aligned}
u_{t} & \leq(r-\lambda w) \cdot u \\
& \leq\left(r-\lambda \cdot \frac{r+\alpha}{\lambda}\right) \cdot u \\
& =-\alpha u \text { for all } t \in(0, T),
\end{aligned}
$$


and hence entails

$$
u(t) \leq u_{0} \cdot e^{-\alpha t} \quad \text { for all } t \in(0, T)
$$

Since $u_{0}<\delta$, this proves that the alternative $T<\infty$ is impossible, and that according to (5.9), the inequality $w(t) \geq \frac{r+\alpha}{\lambda}$ is actually valid for all $t>0$, which proves the claim.

\section{Numerical Investigations}

In this section we perform a series of numerical investigations aimed at both validating the theoretical results of previous sections as well as exploring further system properties. We begin by validating the key restriction on the size of the initial matrix density, (1.3) of Theorem 1.2, for convergence of the cancer invasion model to the chemotaxis growth model. Note that the direct consequence is that $w(x, t) \rightarrow 0$ as $t \rightarrow \infty$, with the cell population replacing the matrix and correlating to tumor expansion.

The numerical scheme adopts a Method of Lines approach in which the equations are first discretized in space on a uniform mesh (of spacing $\Delta x$ ), and the subsequent system of ODEs are then integrated in time. Discretization of the diffusion terms is performed with a central differencing scheme, while the advective term is discretized using a high-order upwinding scheme with flux-limiting imposed to maintain positivity (e.g. see Ref. 7). We use the RowMAP stiff-systems integrator $^{18}$ to integrate the ODEs. Except where specified, we set error tolerances of $10^{-8}$ in ROWMAP. Verification of the scheme has been performed through varying $\Delta x$, error tolerances and using an independent (fully explicit) time-stepping scheme for a representative set of numerics. The qualitative behavior of the system has also been independently confirmed using the MATLAB internal PDE solver (PDEPE).

\subsection{Uniform initial matrix densities}

In Fig. 2 we plot two simulations for the same cell/chemoattractant initial conditions $\left(u_{0}(x)=v_{0}(x)=0.001 e^{-x^{2}}\right)$ but different (constant) initial matrix densities. In (a) we set $w(x, 0)=0.975$ and, under the parameter set in Fig. 1(b), we have $r=\lambda=1$ and hence $\left\|w_{0}\right\|_{L^{\infty}(\Omega)}<\frac{r}{\lambda}$. Consequently, the conditions of Theorem 1.2 are satisfied and we expect convergence with the chemotaxis model. Simulations clearly indicate this: the cell population grows and expands throughout the domain, degrading and replacing the matrix. In (b) we now set $w(x, 0)=1.025$ and $(1.3)$ of Theorem 1.2 is not satisfied. While a degree of matrix degradation occurs in the vicinity of the initial cell mass, the degradation is insufficient to allow the population to grow further. Instead the cell population collapses to zero and no tumor expansion is observed.

We next provide a more rigorous test of Theorem 1.2 by examining its validity over a wider spectrum of initial conditions. To this end, we consider the following 


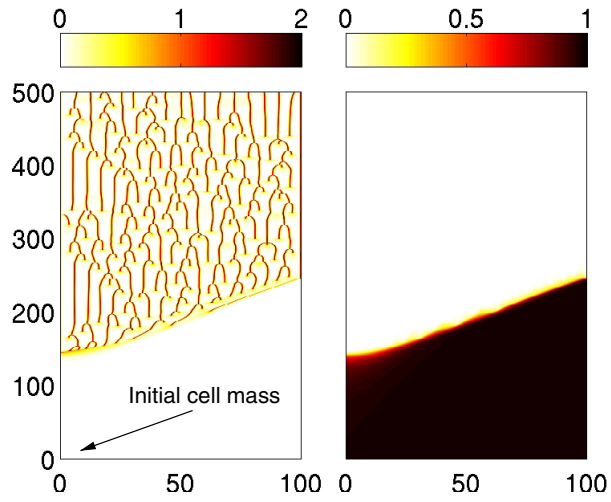

(a)

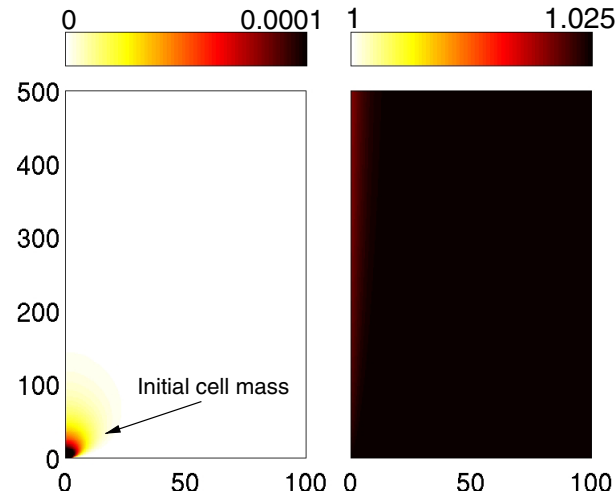

(b)

Fig. 2. Two numerical simulations illustrating the validity of the inequality (1.3) in Theorem 1.2. (a) Space-time density maps showing (left) $u(x, t)$ and (right) $w(x, t)$, with density scales above each frame. Initially $w(x, 0)=0.975, u(x, 0)=0.001 e^{-x^{2}}$. Condition (1.3) is satisfied and the matrix is degraded everywhere. The behavior evolves to that of the logistic growth chemotaxis model (with spatio-temporal patterning for the parameter set under investigation). (b) Spacetime density maps showing (left) $u(x, t)$ and (right) $w(x, t)$ for $w(x, 0)=1.025, u(x, 0)=v(x, 0)=$ $0.001 e^{-x^{2}}$. Here (1.3) is not met. Instead, the cell density evolves to zero and the matrix density remains above 1. Parameters, numerical code and discretization details are as in Fig. 1(b), with simulations performed on a domain $[0,100]$.

two sets:

$$
\begin{array}{lll}
u_{0}(x)=u^{*}, & v_{0}(x)=u^{*}(1+\epsilon(x)), & w_{0}(x)=w^{*}, \\
u_{0}(x)=u^{*} e^{-x^{2}}, & v_{0}(x)=u^{*} e^{-x^{2}}, & w_{0}(x)=w^{*},
\end{array}
$$

where $u^{*}$ and $w^{*}$ are constants and $\epsilon(x)$ denotes a small (1\%) spatially random perturbation.

In Fig. 3 we plot the results of a numerical sweep across $u^{*}-w^{*}$ parameter space for each set of initial conditions. At each value of $u^{*}$ we iterate $w^{*}$ in steps of 0.01 up to $w^{*}=5$ and determine whether the solution converges to the logistic growth chemotaxis model (i.e. $w(x, t) \rightarrow 0$ ) or whether the cell population is wiped out $(u(x, t) \rightarrow 0)$. We augment this plot with the constant line indicating $\frac{r}{\lambda}$ from the restriction (1.3). We note that the validity of Theorem 1.2 is always upheld: for initial conditions lying on/below the line $w^{*}=\frac{r}{\lambda}$ we observe convergence with the chemotaxis model. We further note, however, that while for small $u^{*}$ the bound provides a good approximation for when tumor growth will take place, for larger $u^{*}$ it loses its predictive potential. In this region the initial cell population is sufficiently large that significant matrix degradation takes place, resulting in $w(x, t)$ decreasing below $\frac{r}{\lambda}$ in some regions of the domain. The cell density subsequently increases in these regions, fuelling further matrix degradation. The eventual result is complete matrix degradation and convergence to the logistic growth model. These results echo our estimates in Lemma 3.4, which shows that a large enough $\int_{\Omega} u$ will still 




Fig. 3. Numerical simulations evaluating the validity of Theorem 1.2 under varying initial conditions, see text for details. Each of the lines mark the borders between regions in which the solution converges to the chemotaxis model (i.e. $w(x, t) \rightarrow 0$ ) or whether the cell population is wiped out $(u(x, t) \rightarrow 0)$. Solid line with circles - (IC1); dashed line with squares - (IC2); dotdashed line - inequality (1.3) in Theorem 1.2. For all initial conditions, the inequality holds true: provided $w^{*} \leq 1\left(=\frac{r}{\lambda}\right)$ we have $w(x, t) \rightarrow 0$. For all simulations, model parameters and numerical details are as in Fig. 1(b), solved for $x \in[0,25]$.

lead to matrix degradation, even if (1.3) is not satisfied. However, it is impossible to derive an exact condition such that $\int_{\Omega} u$ will remain large enough for sufficiently long to lead to full matrix degradation.

\subsection{Varying initial matrix densities}

While Theorem 1.2 provides an indication on the matrix density for which we expect convergence to the chemotaxis model, the dependence on $\inf _{x \in \Omega} w_{0}(x)$ is less apparent. We investigate this here through opposite numerical simulations under varying initial matrix density. Specifically, we choose $w_{0}(x)$ to be of the form

$$
w_{0}(x)=\frac{w^{-}+w^{+}}{2}+\frac{w^{+}-w^{-}}{2} \tanh c\left(x^{*}-x\right) .
$$

The above defines a smooth step from $w^{+}$to $w^{-}$centered around $x=x^{*}$, where we choose the center of the domain $x^{*}=50$ in our numerical experiments. Clearly, for sufficiently large $c,\left\|w_{0}\right\|_{L^{\infty}(\Omega)} \simeq w^{+}$and $\inf _{x \in \Omega} w_{0}(x) \simeq w^{-}$. In all simulations we use the parameter set from Fig. 1(b) and hence $\frac{r}{\lambda}=1$.

We begin by considering $u_{0}(x)$ and $v_{0}(x)$ as in (IC1) with $u^{*}=0.001$. In Fig. 4(a) we set $w^{-}=1.05$ and $w^{+}=1.5$ and hence $\left\|w_{0}\right\|_{L^{\infty}(\Omega)}>\inf _{x \in \Omega} w_{0}(x)>1$. Clearly inequality (1.3) is not met and we cannot definitively expect convergence. Simulations here reveal decay of the cell population to zero with the matrix density 


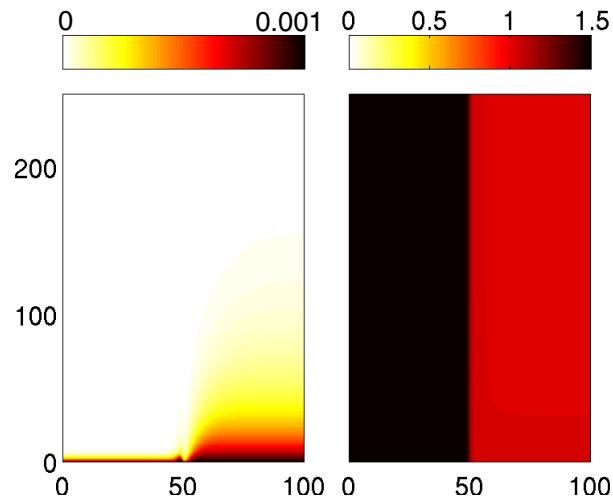

(a)

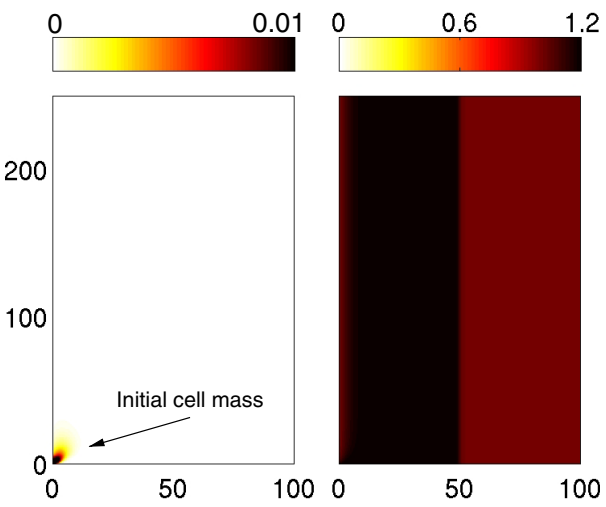

(c)
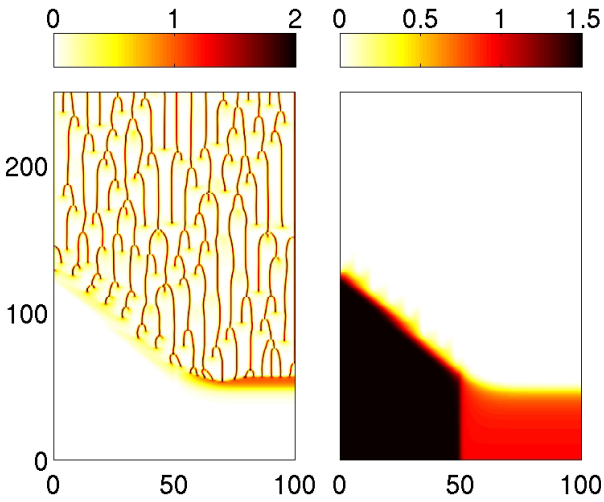

(b)

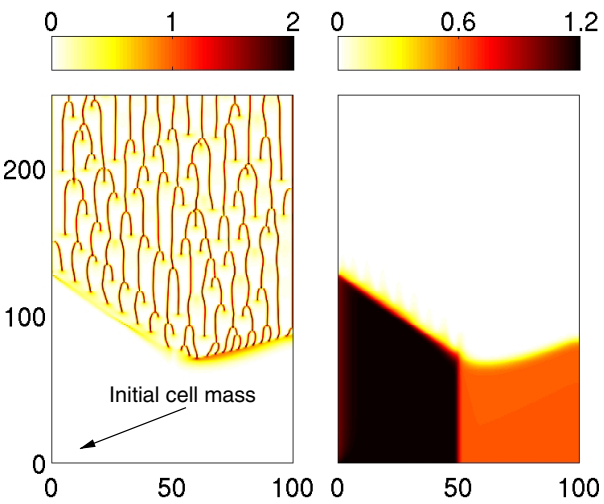

(d)

Fig. 4. Numerical simulations under varying initial matrix density. In each of (a)-(d) we plot the space-time density map of (left) $u(x, t)$ and (right) $w(x, t)$, with density scales above each frame. Initial matrix density as given in (6.1) with $c=1, x^{*}=50$ and (a) $w^{+}=1.5, w^{-}=1.05$; (b) $w^{+}=1.5, w^{-}=0.95$; (c) $w^{+}=1.2, w^{-}=1.01$; (d) $w^{+}=1.2, w^{-}=0.6$. Initially cells are either distributed uniformly, as in (a)-(b), or are concentrated at the $x=0$ boundary (c)-(d), see text for details. For all simulations, model parameters and numerical details are as in Fig. 1(b), solved for $x \in[0,100]$.

remaining above 1 for all $x$. In Fig. 4(b) we now set $w^{-}=0.95$ and $w^{+}=1.5$, i.e. $\left\|w_{0}\right\|_{L^{\infty}(\Omega)}>1>\inf _{x \in \Omega} w_{0}(x)$. While (1.3) is still not satisfied, in the region of low matrix density the cell population grows, fuelling further matrix degradation. The cells simultaneously move into and degrade the region of higher matrix density, eventually resulting in $w \rightarrow 0$ and convergence to the chemotaxis model.

We examine similar simulations for an initially concentrated cell mass in the region of higher matrix density, taking $u_{0}(x)$ and $v_{0}(x)$ from (IC2) with $u^{*}=0.1$. For Fig. 4(c) we set $w^{-}=1.01$ and $w^{+}=1.2$. As in Fig. 4(a), the cell density drops to zero and the matrix density is not sufficiently degraded to allow tumor expansion to occur. For Fig. $4(\mathrm{~d})$ we set $w^{-}=0.6$ and $w^{+}=1.2$. While matrix 
is not sufficiently degraded in the region of higher matrix density, here the cell dispersal terms allow transport of a small fraction into the lower matrix density region. In this region, $w<1$ and the cell population begins to grow and degrade the matrix. Eventually we observe tumor expansion and convergence to the logistic growth chemotaxis model.

In summary, these and further simulations (data not shown) suggest that (1.3) is only a sufficient condition for convergence. While it provides a valid restriction on the initial matrix density for tumor expansion and convergence to the chemotaxis model there are also numerous cases where (1.3) is not true and we still get convergence. In particular, the simulations indicate that for situations in which $\lambda \inf _{x \in \Omega} w_{0}(x)<\frac{r}{\lambda}$, but $\lambda\left\|w_{0}\right\|_{L^{\infty}(\Omega)}>r$ we also observe convergence. Furthermore, as indicated in Fig. 3 it is even possible to observe convergence for $\inf _{x \in \Omega} \lambda w_{0}(x)>r$, provided the initial cell population is sufficiently large.

\subsection{Sensitivity to initial data}

A key observation in Ref. 12 was a sensitivity dependence to initial conditions for certain classes of irregular spatio-temporal patterns, a finding suggested to indicate spatio-temporal chaos in the chemotaxis model. We demonstrate the extension of this property to the tumor invasion model in Fig. 5. In (a) simulations are initiated according to (IC2) under $u^{*}=0.01$ and $w^{*}=1$; as expected from the above findings, we observe degradation of the matrix and convergence to spatio-temporal patterning. In (b) we apply a small $(0.1 \%)$ random spatial perturbation to the



(a)

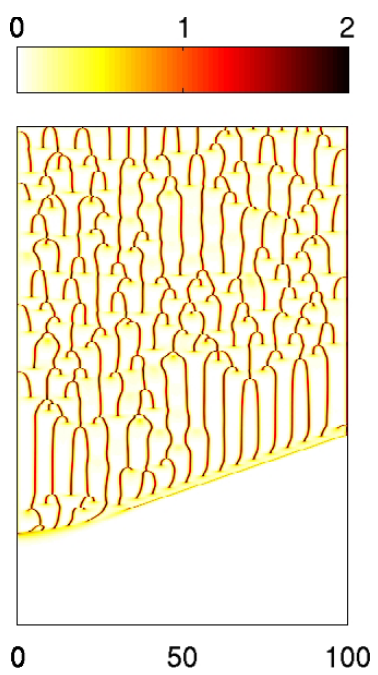

(b)


(c)

Fig. 5. Simulations demonstrating sensitivity dependency to initial conditions, see text for details. For all simulations, model parameters and numerical details are as in Fig. 1(b), solved for $x \in[0,100]$. 
initial condition for $u_{0}(x)$. In the initial tumor invasion phase, the behavior is almost identical. However, following convergence to spatio-temporal irregularity we eventually observe divergence of the solutions, illustrated in the difference of the solutions in (c).

\subsection{Incorporating matrix regeneration}

In the model studied here only matrix degradation was considered, however in many instances the matrix may undergo repair as a part of the normal physiological response to damage. The models of Chaplain and coworkers have accounted for matrix repair through additional terms incorporated into the equation for $w$ in (1.1). Permitting matrix repair significantly complicates the derivation of conditions for which matrix degrades to zero. We investigate this case numerically, modifying
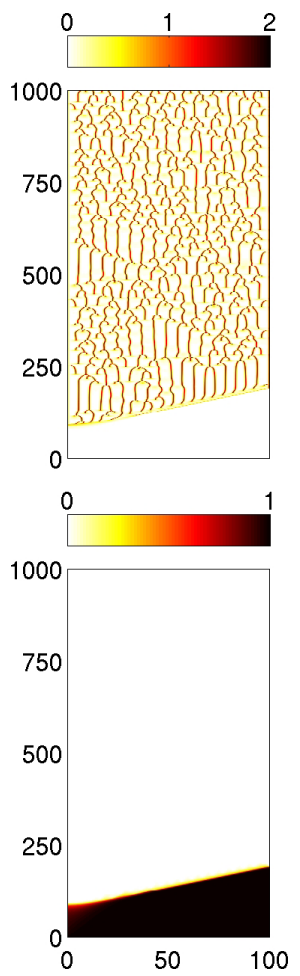

(a)
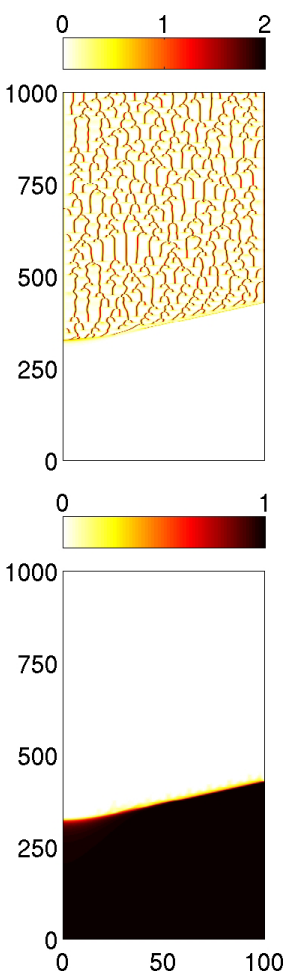

(b)



(c)

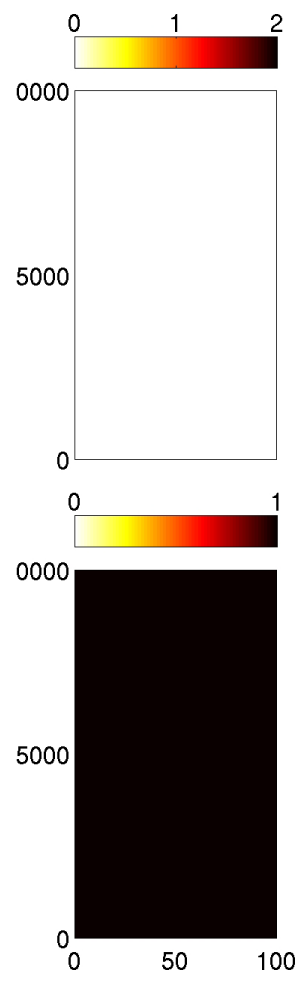

(d)

Fig. 6. Numerical simulations of the cancer invasion model (1.1) under inclusion of matrix regeneration. Specifically, the equation for $w$ is augmented on the right-hand side with an additional term $\mu_{2} w(1-w)$. Each frame plots the space-time density map for (top rows) cell density $u$ and (bottom rows) matrix density $w$. The parameter values are as in Fig. 1(b) with, for the additional terms, (a) $\mu_{2}=0$; (b) $\mu_{2}=0.1$; (c) $\mu_{2}=0.2$ and (d) $\mu_{2}=1.0$. Initial conditions and all other numerical details as for simulations of the cancer invasion model are as in Fig. 1(b). 
the equation for $w$ in (1.1) to

$$
w_{t}=\mu_{2} w(1-w)-\rho v w,
$$

as considered in Refs. 3 and 4. In these illustrative simulations we increase the values of $\mu_{2}$, corresponding to an increasingly rapid matrix repair response. In Fig. 6 we compare results from simulations for the above kinetics. For $\mu_{2}$ close to zero similar behavior is observed to system (1.1), although for all values $\mu_{2}>0$ investigated it is noted that the matrix does not (over the timescale of numerics) completely degrade to zero and the rate of tumor invasion is delayed. For larger values of $\mu_{2}$ the matrix repair exerts an increasingly strong impact on the dynamics, and eventual loss of pattern formation.

\section{Conclusions}

The models of Chaplain et al. ${ }^{3,4,2}$ have been designed to model the degradation and infiltration of a healthy ECM-dominated tissue by an invasive cancerous population through haptotactic and chemotactic guided migration. The original model ${ }^{3}$ was developed according to the principle interactions involved in the urokinase plasminogen system and consists of five coupled nonlinear partial differential equations, while the model analyzed in the present paper was based on a reduced system of three partial differential equations studied in Ref. 4. The models clearly show tumor invasion into healthy tissue, followed in certain regions of parameter space by complicated spatio-temporal patterning. The principal aim of the current paper is to determine the origin of these complicated dynamics. Noting the similarity in behavior between the tumor invasion model and a simpler chemotaxis model, shown in Ref. 12 to exhibit spatio-temporal chaos, we derived conditions under which the two models converge. Thus, at least for low levels of ECM regeneration, we conclude that the organizing center for these spatial patterns is a chemotaxis model with logistic reproduction terms. While we should note that the aim of the present paper has been focused towards a deeper analytical understanding of a model rather than application dominated, it is worth highlighting that these spatial patterns qualitatively replicate the complicated morphologies of certain forms of invasive tumor. Identifying such spatio-temporal dynamics of tumor morphology in vitro and in vivo would be extremely interesting.

It is worth stressing that a merely superficial examination of the third equation of (1.1)

$$
w_{t}=-\rho w v
$$

immediately suggests that $w$ collapses to zero and results in the chemotaxis system, suggesting that the results here could be construed as somewhat obvious in nature. However, the key point is that such blind intuition can be misleading and that there are clear cases, identified numerically and analytically, where convergence does not occur. In fact we have only identified a sufficient condition (1.3) under 
which convergence occurs and our numerical simulations reveal clearly that this condition is not optimal. If (1.3) is not satisfied, then solutions might or might not converge. Lemma 3.4 gives an indication that for large enough $\int_{\Omega} u$ convergence can be expected. The simulations also show a dichotomy; in all cases studied, we either obtain convergence towards the chemotaxis model, or convergence of $u$ to zero. In Theorem 1.4 we could at least show that $\liminf _{t \rightarrow \infty}\|u(\cdot, t)\|_{L^{1}(\Omega)}$ is zero, if there is no convergence.

To obtain our results, we needed to make certain assumptions. Our estimates are strictly one-dimensional, and a corresponding result in higher dimensions would be desirable. Secondly, and crucially from a biological perspective, we have had to remove the self-renewal term for the ECM. Simulations suggest that with ECM remodeling, we still observe the same dynamics provided the repair rate is not too strong and a detailed analysis of this case is left for future work. We also note that the present paper highlights the non-trivial task of scratching deeper under the surface of models being developed to describe complicated dynamics. As biological models become more involved, incorporating more components and characteristics of in vivo tissues, it will become increasingly important to find the scenarios under which their behavior can be understood in terms of simpler and more established systems.

\section{Acknowledgments}

K.J.P. acknowledges research support from Leverhulme Trust (Research Fellowship RF-2011-045), and T.H. acknowledges research support from NSERC.

\section{References}

1. M. Aida, T. Tsujikawa, M. Efendiev, A. Yagi and M. Mimura, Lower estimate of the attractor dimension for a chemotaxis growth system, J. London Math. Soc. 74 (2006) 453-474.

2. V. Andasari, A. Gerisch, G. Lolas, A. South and M. A. J. Chaplain, Mathematical modeling of cancer cell invasion of tissue: Biological insight from mathematical analysis and computational simulation, J. Math. Biol. 63 (2011) 141-172.

3. M. A. J. Chaplain and G. Lolas, Mathematical modelling of cancer cell invasion of tissue: The role of the urokinase plasminogen activation system, Math. Models Methods Appl. Sci. 15 (2005) 1685-1734.

4. M. A. J. Chaplain and G. Lolas, Mathematical modelling of cancer invasion of tissue: Dynamic heterogeneity, Net. Hetero. Med. 1 (2006) 399-439.

5. D. Hanahan and R. A. Weinberg, Hallmarks of cancer: The next generation, Cell 144 (2011) 646-674.

6. T. Hillen and K. J. Painter, A user's guide to PDE models for chemotaxis, J. Math. Biol. 58 (2009) 183-217.

7. W. Hundsdorfer and J. G. Verwer, Numerical Solution of Time-Dependent AdvectionDiffusion-Reaction Equations, Springer Series in Computational Mathematics, Vol. 33 (Springer-Verlag, 2003).

8. G. Liţcanu and C. Morales-Rodrigo, Asymptotic behaviour of global solutions to a model of cell invasion, Math. Models Methods Appl. Sci. 20 (2010) 1721-1758. 
9. A. Marciniak-Czochra and M. Ptashnyk, Boundedness of solutions of a haptotaxis model, Math. Models Methods Appl. Sci. 20 (2010) 449-476.

10. E. Nakaguchi and M. Efendiev, On a new dimension estimate for the global attractor for chemotaxis-growth systems, Osaka J. Math. 45 (2008) 273-281.

11. K. Osaki, T. Tsujikawa, A. Yagi and M. Mimura, Exponential attractor for a chemotaxis-growth system of equations, Nonlinear Anal. 51 (2002) 119-144.

12. K. J. Painter and T. Hillen, Spatio-temporal chaos in a chemotaxis model, Physica D 240 (2011) 363-375.

13. Y. Tao, Global existence of classical solutions to a combined chemotaxis-haptotaxis model with logistic source, J. Math. Anal. Appl. 354 (2009) 60-69.

14. Y. Tao and M. Wang, Global solution for a chemotactic-haptotactic model of cancer invasion, Nonlinearity 21 (2008) 2221-2238.

15. Y. Tao and M. Wang, A combined chemotaxis-haptotaxis system: The role of logistic source, SIAM J. Math. Anal. 41 (2009) 1533-1558.

16. Y. Tao and M. Winkler, A chemotaxis-haptotaxis model: The roles of porous medium diffusion and logistic source, SIAM J. Math. Anal. 43 (2011) 685-704.

17. C. Walker and G. F. Webb, Global existence of classical solutions for a haptotaxis model, SIAM J. Math. Anal. 38 (2007) 1694-1713.

18. R. Weiner, B. A. Schmitt and H. Podhaisky, Rowmap - A row-code with Krylov techniques for large stiff ODEs, Appl. Numer. Math. 25 (1997) 303-319.

19. M. Winkler, Aggregation vs. global diffusive behavior in the higher-dimensional Keller-Segel model, Differential Equations 248 (2010) 2889-2905.

20. M. Winkler, Boundedness in the higher-dimensional parabolic-parabolic chemotaxis system with logistic source, Commun. Partial Differential Equations 35 (2010) 15161537. 